\title{
An ethnobotanical survey of wild edible plants used by the Yi people of Liangshan Prefecture, Sichuan Province, China
}

\author{
Jing Wang ${ }^{1}$, Barnabas C. Seyler ${ }^{2^{*}}$ (D, Tamara Ticktin ${ }^{3}$, Yonggang Zeng ${ }^{1}$ and Kede Ayu ${ }^{1,4}$
}

\begin{abstract}
Background: Due to historical perceptions of Liangshan Yi Autonomous Prefecture (Sichuan Province, China) as being a violent place, and due to its rugged terrain, cultural differences, and relative inaccessibility, few researchers have conducted in-depth ethnobotanical investigations in Liangshan. But wild edible plants (WEPs) are widely consumed by the Yi people of Liangshan, and their associated ethnobotanical knowledge remains relatively unknown, especially outside of China. This study aimed to (1) investigate the WEPs used by the Liangshan Yi, (2) document the traditional knowledge held about these plants, (3) analyze their special preparation methods and consumption habits, and (4) identify species with important cultural significance to the Liangshan Yi.

Methods: During 2016-2017, 396 Yi individuals were interviewed in 1 county-level city and 6 counties across Liangshan. Prior informed consent was obtained, and multiple ethnographic methods were utilized, including direct observation, semi-structured interviews, key informant interviews, informal discussions, and field visits. Market surveys were conducted in April, July, and August 2017 by interviewing 38 Yi merchants selling WEPs in 6 Liangshan traditional markets. We collected information about the parts consumed, preparation methods, consumption habits, growth pattern of species, collection months, market prices, and other uses of WEPs. Use values (UVs) were calculated to analyze the relative cultural importance of each WEP.
\end{abstract}

Results: In total, 105 plant species belonging to 97 genera and 62 families were recorded. Rosaceae was the family with the largest number of species (14), and herbs (58 species) were the dominant growth form reported. Fruits (34 species), roots (21 species), and tender shoots (20 species) were the primary plant parts used for snacking and cooking. There were 6 main preparation and consumption methods of WEPs reported, ranging from primary food, famine food, snack, spice, culinary coagulant, and medicine, among a few other uses. The Liangshan Yi mainly collect WEPs from March to October, seldom collecting from November to February. There were 35 species of WEPs sold in the markets we visited in Liangshan. The price of medicinal plants was much higher than the price of food and fruits. In total, we documented 49 species of edible medicinal plants in Liangshan, accounting for $44.7 \%$ of all WEPs. They can be used for treating 27 medical conditions, including cough, diarrhea, injury, and headaches. The plants with the highest UVs were Berberis jamesiana (1.92), Pyracantha fortuneana (1.87), and Artemisia capillaris (1.44) indicating that these species are the most commonly used and important to the Liangshan Yi's traditional life and culture.

(Continued on next page)

\footnotetext{
* Correspondence: bseyler@scu.edu.cn

2Department of Environment, Sichuan University, Chengdu 610065, China

Full list of author information is available at the end of the article
}

(c) The Author(s). 2020 Open Access This article is distributed under the terms of the Creative Commons Attribution 4.0 International License (http://creativecommons.org/licenses/by/4.0/), which permits unrestricted use, distribution, and reproduction in any medium, provided you give appropriate credit to the original author(s) and the source, provide a link to the Creative Commons license, and indicate if changes were made. The Creative Commons Public Domain Dedication waiver (http://creativecommons.org/publicdomain/zero/1.0/) applies to the data made available in this article, unless otherwise stated. 
(Continued from previous page)

Conclusions: The traditional knowledge of WEPs from the accumulated experience of the Yi people's long period residing in Liangshan reflects the cultural richness of the Yi and the plant diversity of the region. Future research on the nutrition, chemical composition, and bioactivity of the WEPs are needed. Some species with high medicinal value but with sharp wild population decline should be surveyed for resource assessment, conservation, and domestication potential.

Keywords: Liangshan Yi Autonomous Prefecture, Yi people, Wild edible plants, Use values, Ethnobotany

\section{摘要}

背景:由于凉山彝族自治州(中国四川省)地形崎岖、交通不便，文化制约、历史上民风彪悍，很少有学者对凉山 地区进行深入的民族植物学调查。凉山彝族采集食用多种野生食用植物(WEPs)，但其相关的民族植物学知识 鲜为人知, 国外对此更是无从知晓。本研究的目的是:(1)对凉山撛族所食用的野生植物进行系统的研究; (2)记 录这些植物的传统知识; (3)分析其特殊的制备方法和消费习惯; (4)识别对凉山彝族有重要文化意义的物种。 方法:在2016-2017年间, 对凉山州1个县级市和6个县的396名彝族人进行了访谈。采用了多种人类学方法, 包括 直接观察、半结构式访谈、关键人物访谈、非正式讨论和田野调查。市场调查于2017年4月、7月和8月进行， 调查了凉山州6个传统集市，并访问了38位彝族商贩。我们收集这些野生食用植物的利用部位、制备方 法、利用方式, 生长模式、采集月份、市场价格和其他用途的相关信息。采用使用价值指数(UVS)来分析每个 野生食用植物的的相对文化重要性。

结果:本研究共记录野生食用植物 105种，隶属于97属62科。其中蓄薇科植物种类最多(14种), 草本植物占优 势(58种)。食用部位主要是果实(34种)，根(21种)和嫩枝(20种)，果实多作为零食食用，根和嫩枝多需要进行烹杄 后食用。凉山彝族制备和利用野生食用植物的方法可分为六类，即，日常饮食、饥荒食物、零食、调味

品、食用凝固剂和药用植物。凉山彝族主要在3月至10月采集野生食用食物, 很少在11月至来年2月进行采集 工作。调查发现有35种野生食用植物用于出售。药用植物的价格远高于食物和野果的价格。本研究共记录 凉山地区食用药用植物49种，占记录的野生食用植物总种数的 $44.67 \%$, 它们可用于治疗 27 种疾病，包括咳 嗽、腹泻、受伤和头痛。使用价值(UVs)最高的植物是川滇小檗(1.92)、火棘(1.87)和茵陈蒿(1.44)，表明这些植 物是凉山彝族传统生活和文化中最常用和最重要的种类。

结论凉山彝族利用野生食用植物的传统知识源于在当地长期居住积累的生活经验，反映了凉山彝族文化的丰 富性和该地区植物的多样性。未来可对这些野生食用植物的的营养成分、化学成分和生物活性进行进一步 的研究; 对一些具有较高药用价值但野生种群数量急剧下降的物种应进行资源评估、保护和驯化潜力调查研 究。

\section{Introduction}

Adapting to continued human population growth and global climate change requires a diversity of food plants to ensure a safe and resilient food supply [1-7]. In particular, wild edible plants (WEPs) are of great significance in maintaining the productivity and stability of traditional agro-ecosystems [5, 8]. In times of famine and scarcity, these sources of nutrients and healthpromoting compounds have received heightened attention in rural and suburban areas $[9,10]$. WEPs remain essential components of the diets for many people in developing countries, especially in periods of seasonal food shortage [11]. Consequently, conserving WEPs is necessary to ensure the ongoing supply of diverse genetic resources that are critical to global food security $[2,4,5,12]$.

As living standards rise, there is also an increasing global demand for healthier and safer food [13]. Compared with cultivated vegetables, WEPs require less maintenance, are not dependent on chemical fertilizers or pesticides, and are richer sources of micronutrients [5]. Some
WEPs have also been described as "functional foods" because they contain physiologically active ingredients capable of providing health benefits beyond basic nutrition [10]. Multiple authors have noted a continuum in many cultures between "food" and "medicine" plants, with species initially selected as medicine later used primarily for food (or vice versa), and the concepts of food and medicine themselves are infrequently differentiated, instead taken together as synonymous with "healthy eating" [9, 14-17]. Furthermore, whether a particular plant is perceived as a food, medicine, or even poison often depends on the part used or quantity ingested, as well as how and when it is collected/prepared $[9,16]$.

Since local cultures select edible species over time based on many years of experience, traditional ethnobotanical knowledge and associated practices about WEPs are highly dependent on the local context [10]. It is, therefore, increasingly important to carry out systematic ethnobotanical investigations to document WEPs utilized in rural communities and by the socio-cultural 
groups that are dependent upon them [18]. Fortunately, in recent decades, focused studies of WEPs have proliferated worldwide, including in Africa [6, 19, 20], South Asia [4, 21], East Asia [5, 22, 23], Europe [10, 24], North America [17], South America [7, 11], and Oceania [25]. Nevertheless, the WEPs utilized by many unique sociocultural groups in each of these diverse geographic regions remain understudied. Yet, in addition to documenting WEPs around the world, studies should also strive for theoretical rigor by testing hypotheses associated with the use, selection, and perception of WEPs in the local communities [26].

Liangshan Yi Autonomous Prefecture (Liangshan) is a mountainous rural jurisdiction in southwest Sichuan Province, China. It is the single largest settlement area for the Yi minority people in China, being the primary home of the most populous and geographically most widely distributed branch of Yi [27, 28]. For many centuries, Liangshan was considered by outsiders to be an especially dangerous place, with violent clashes and open warfare between the $\mathrm{Yi}$ and Han Chinese populations [29]. Liangshan was the last significant region of China to resist Communist control, so the people living there largely governed their own affairs until the Communist penetration of the area began in the 1950s. Before the 1956 reforms, the Yi people of Liangshan maintained a rigid slave-based feudal society, raiding neighboring Han communities and subjugating travelers as slaves. Despite the formal abolition of the system in the 1950s, the hierarchical classes, associated values systems, and other unique cultural characteristics have remained, largely intact, to this day [30]. Consequently, due to external perceptions about its remoteness, rugged terrain, cultural considerations, and relative inaccessibility, few ethnobotanists have conducted in-depth investigations in Liangshan.

Of note, few studies have specifically analyzed the WEPs harvested by the Liangshan Yi, and the only indepth studies of this kind (all published in Chinese) have documented plants used for food by the Yi in a single county of Liangshan [31], as well as plants used for dye [32] and folk customs [33]. The richness of Liangshan's traditional culture and biodiversity warrants deeper investigations. Therefore, this study sought to identify the WEPs used by Liangshan's Yi people, document their uses, the plant parts used, and the traditional knowledge held about these plants. We also sought to assign plant use value scores to the WEPs utilized by the Liangshan Yi to identify the culturally most important taxa, which will help prioritize plants for conservation purposes. Based on the composition of the local flora, and because family tends to be a strong predictor of plant use value [26, 34], we hypothesized that (1) certain families (Compositae, Lamiaceae, Rosaceae) would have more species with higher use values as a result of their (a) greater abundance in Liangshan's native flora and (b) global importance for food crop species. Although other globally important food-crop families (e.g., Solanaceae) are now commonly cultivated by the Liangshan $\mathrm{Yi}$, they are relatively less common among the local flora. We also hypothesized that (2) fruit would be the plant part most frequently used, due to the local abundance of edible fruiting trees in the local flora; (3) most plants would be harvested during summer and fall, due to the seasonality of fruit maturation; and (4) given that there is no clear distinction in many cultures between the concepts of food and medicine, some WEPS would also have medicinal value.

\section{Materials and methods}

\section{Liangshan's ecology and climate}

Liangshan Prefecture encompasses $60,423 \mathrm{~km}^{2}$ and, located in the southwest of Sichuan Province, lies between $26^{\circ} 03^{\prime}$ to $29^{\circ} 18^{\prime} \mathrm{N}$ latitude and $100^{\circ} 03^{\prime}$ to $103^{\circ} 52^{\prime} 2 \mathrm{E}$ longitude. The region has a subtropical monsoon climate, with warm winters, dry springs, and ample sunshine year-round. The rainy monsoon conditions with passing clouds moderate the summer temperatures as well, with an average annual temperature of $16-17^{\circ} \mathrm{C}$. To the east, west, and south, Liangshan is surrounded on three sides by the Jinsha River, and, to the north, it is bounded by the Dadu River, thus forming a relatively closed geographical unit. With the Greater and Lesser Xiangling Mountains as the boundary, there is a distinct contrast of climates in Liangshan with dryer conditions to the south and west and moister conditions to the north and east.

Liangshan also constitutes the northern section of the Hengduan Mountain Range, which serves as a topographic bridge between the Sichuan Basin and the Yunnan-Guizhou Plateau [35], forming the core area of the Eastern Himalayan Biodiversity Hot Spot [36]. Consequently, Liangshan is dominated by mountains and plateaus, accounting for more than $90 \%$ of the entire area, with the remaining $10 \%$ being hills, basins, and plains. The altitude ranges from $325 \mathrm{~m}$ below sea level up to $5958 \mathrm{~m}$ above sea level. These elevational extremes, with warmer temperatures prevailing at lower altitudes and cooler conditions at higher altitudes, also create a wide diversity of microclimates in close proximity, with a great abundance of plant species and high endemism. The vegetation cover of Liangshan includes more than 2 million hectares of woodlands and grasslands with more than 4000 plant species [37]. Due to its rich plant resources, the people living in the region for generations, particularly the $\mathrm{Yi}$, have accumulated a wealth of ethnobotanical knowledge [33, 38]. 


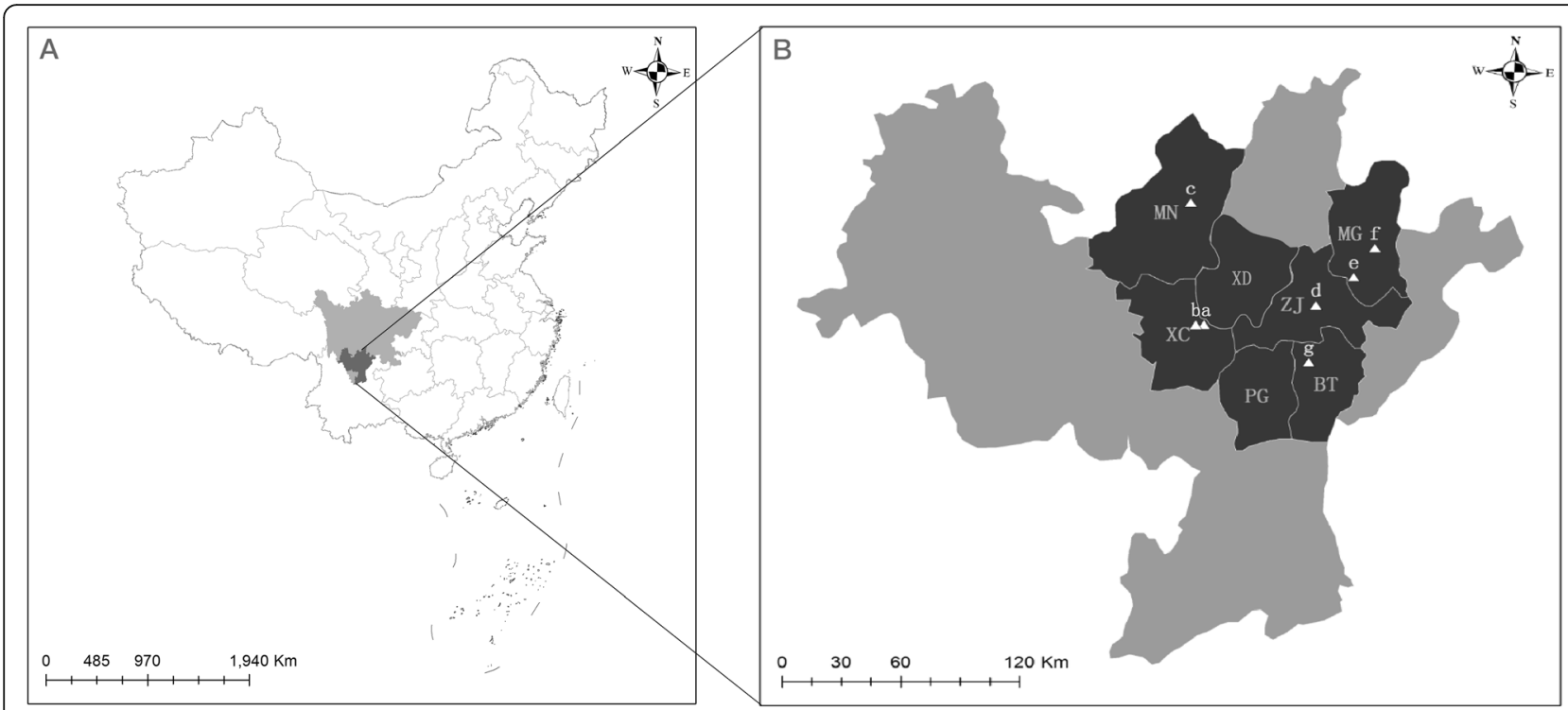

Fig. 1 Location of the study areas. a Location of Liangshan Prefecture (dark gray) within Sichuan Province (light gray) and China (white). b Location of the field survey jurisdictions (dark gray) within Liangshan (light gray), with market locations indicated by triangles. BT, Butuo County; MG, Meiggu County; MN, Mianning County; PG, Puge County; XC, Xichang City; XD, Xide County; Z, Zhaojue County. (a) Xichang Binhe Market; (b) Chang'an Village Farmers Market, Xichang City; (c) Mianning County Farmers Market; (d) Zhaojue Comprehensive Farmers Market; (e) Jiukou Township Market, Meigu County; (f) Meigu County Farmers Market; (g) Butuo County Farmers Market

\section{Liangshan demographics, language, and study site locations}

The Yi comprise the seventh largest ethnic group in China, with a total population of about 9 million people primarily spread across the southwest Chinese provinces of Yunnan, Sichuan, and Guizhou. The Yi account for the majority of Liangshan's total population, with the 2016 census indicating that of Liangshan's 5,117,825 people, 2,647,791 were Yi (51.7\%), followed by the Han (44.8\%), Tibetan (1.41\%), Mongolian (0.64\%), and Hui

Table 1 Study site locations

\begin{tabular}{|c|c|c|c|c|c|c|c|c|c|}
\hline $\begin{array}{l}\text { County/ } \\
\text { city } \\
\text { name }\end{array}$ & $\begin{array}{l}\text { Fieldwork } \\
\text { months }\end{array}$ & $\begin{array}{l}\text { Area } \\
\text { (ha) }\end{array}$ & $\begin{array}{l}\text { Total } \\
\text { population }\end{array}$ & $\begin{array}{l}\text { Yi } \\
\text { population }\end{array}$ & $\begin{array}{l}\text { Percentage } \\
\text { (Yi/total) }\end{array}$ & $\begin{array}{l}\text { Annual per } \\
\text { capita } \\
\text { income } \\
\text { (RMB) }\end{array}$ & $\begin{array}{l}\text { Participants } \\
\text { (F/M) }\end{array}$ & $\begin{array}{l}\text { Market survey location (no. of } \\
\text { participants) }\end{array}$ & $\begin{array}{l}\text { Latitude and } \\
\text { longitude } \\
\text { (market) }\end{array}$ \\
\hline Butuo & $\begin{array}{l}\text { Apr., Jul., Aug., } \\
\text { Oct. } 2017\end{array}$ & 1685 & 191,213 & 184,128 & 96 & $¥ 6386$ & $74(33 / 41)$ & $\begin{array}{l}\text { Butuo County Farmers Market } \\
\text { (6) }\end{array}$ & $\begin{array}{l}\text { 102.8109, } \\
27.7078\end{array}$ \\
\hline Meigu & $\begin{array}{l}\text { Apr., Jul., Aug. } \\
2017\end{array}$ & 2515 & 268,739 & 265,689 & 99 & $¥ 6246$ & $63(32 / 31)$ & $\begin{array}{l}\text { Jiukou Township Market, Meigu } \\
\text { County (3); Meigu County } \\
\text { Farmers Market (5) }\end{array}$ & $\begin{array}{l}103.0252 \\
28.1722 ; \\
103.1307 \\
28.3311\end{array}$ \\
\hline Mianning & $\begin{array}{l}\text { Jul., Aug. 2016; } \\
\text { Apr., Jul., Aug. } \\
2017\end{array}$ & 4422 & 398,071 & 160,098 & 40 & $¥ 11,156$ & $73(38 / 35)$ & $\begin{array}{l}\text { Mianning County Farmers } \\
\text { Market (8) }\end{array}$ & $\begin{array}{l}\text { 102.1761, } \\
28.5518\end{array}$ \\
\hline Puge & $\begin{array}{l}\text { Oct., Nov., Dec. } \\
2016\end{array}$ & 1905 & 198,982 & 165,786 & 83 & $¥ 7454$ & $18(7 / 11)$ & & \\
\hline Xichang & Apr. 2017 & 2657 & 652,947 & 126,493 & 19 & $¥ 13,620$ & $19(5 / 14)$ & $\begin{array}{l}\text { Xichang Binhe Market (8); } \\
\text { Chang'an Village Farmers Market, } \\
\text { Xichang City (2) }\end{array}$ & $\begin{array}{l}\text { 102.2697, } \\
27.8948 \\
102.2284 \\
27.8917\end{array}$ \\
\hline Xide & $\begin{array}{l}\text { Oct., Nov., Dec., } \\
2016 \text {; Jul., Aug. } \\
2017\end{array}$ & 2202 & 229,690 & 208,604 & 91 & $¥ 6347$ & $57(26 / 31)$ & & \\
\hline Zhaojue & Jul., Aug. 2017 & 2702 & 314,461 & 308,555 & 98 & $¥ 6675$ & $92(45 / 47)$ & $\begin{array}{l}\text { Zhaojue Comprehensive Farmers } \\
\text { Market (6) }\end{array}$ & $\begin{array}{l}102.8374 \\
28.0157\end{array}$ \\
\hline & & & & & & Total & \multicolumn{2}{|l|}{$396(186 / 210)$} & \\
\hline
\end{tabular}


(0.42\%), among a few others [39]. In total, Liangshan's population consists of 14 ethnocultural groups with an estimated density of 0.85 people per square kilometer [40].

Administratively, Liangshan is comprised of 16 counties and one county-level city (Xichang City), which serves as the prefectural capital. Prior to 1978, Zhaojue County served as Liangshan's capital. The Liangshan Yi belong to the northern dialect branch of the Yi (Nuosu) language, which is further subdivided into 4 dialects; these are often referred in published literature in Chinese as Sheng-zha (圣乍), Yi-nuo (义诺), Suo-di (所地), and $A-d u$ (阿都) [28, 41, 42]. We chose study sites in six counties and Xichang City, representative of the geographic and cultural diversity of the Liangshan Yi (Fig. 1,

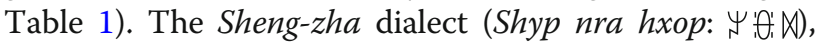
based in Xide County and also widely spoken in Mianning County, Xichang City, and Zhaojue County, is considered the prestige or standard pronunciation for the $\mathrm{Yi}$ Language [41-43]. Butuo County represents the $A-d u$ dialect ( $A$ dur hxop: $y^{\prime}+\mathbb{X}$ ), Meigu County represents the Yi-nио dialect (Yyp nио hxop: $\varepsilon \uparrow X)$, and Puge County represents the Suo-di dialect (Suo ndip hxop: $\pi(1)$ ). All Nuosu words in this paper and supporting materials follow the official Yi language phonetic alphabet, affixing a consonant symbol at the end of certain syllables to represent tones: (1) " $t$ " for a high, flat register, (2) " $\mathrm{x}$ " for a mid to high register (mid-rising tone), and (3) "p" for a low-falling register. There is no mark for the mid-register tone $[28,44]$.

\section{Yi traditional culture}

The ancestors of the Liangshan Yi are believed to be the two ancient tribes of Guhou and Qunei, who once lived in Zizipuwu (Zhaotong area) of what is now Yunnan Province. They moved into Liangshan more than 2000 years ago, and their descendants gradually differentiated into the several tribes that are now spread throughout the Liangshan region [45]. Due to the historic physical and social isolation of the area, the Yi culture of Liangshan is quite different from the Yi living outside of Sichuan (other than a few border areas, including Ninglang County and Zhaotong in northern Yunnan), which have been much more deeply influenced by Han culture and religion [27, 28, 33, 46, 47].

The Liangshan Yi primarily subscribe to polytheistic animism, believing that their ancestors, spirits, and ghosts are able to influence the health of people, the success of their clan, the bounty of the harvest, the fertility of cattle, and the harmony of the community. Rituals serve as the main vehicle for the expression of their beliefs and traditional sentiments, being the primary means for balancing and adjusting the relationships between humans and supernatural beings. The Yi ritual specialists and traditional practitioners are called bimox $(\in \in \bar{\xi})$ and sunyit $\left(f^{-} *\right)$, respectively [48]. Throughout these ceremonies, some WEPs are used for both the ritual concoctions themselves as well as for offerings.

\section{Field survey and data collection}

Between July 2016 and September 2017, we conducted field ethnobotanical surveys in the 7 jurisdictions (6 counties and 1 city) across Liangshan (Table 1). Following snowball sampling methods [49], we interviewed a total of 396 local Yi, of which 195 were female and 201 were male. Participants were between the ages of 12 and 84. The purpose of the study was briefly explained to each, and informed consent was obtained orally. Ethnobotanical data were collected using different ethnographic methods (direct observation, semi-structured interviews, key informant interviews, informal discussions, and field visits) with the assistance of native $\mathrm{Yi}$ language translators. Interviews took place in a location of each participant's choice, often being their homes but sometimes in the field. Following established interview protocols [20, 49-51], participants were first asked to name the WEPs that they gather, then asked follow-up questions about the parts consumed, preparation methods, consumption habits, growth pattern of the species, collection months, and other uses of the WEPs. Demographic variables of each participant were also collected at the end of each interview, including their name, age, sex, level of education, and occupation.

During the investigation, we also conducted market surveys in April, July, and August 2017, interviewing 38 Yi merchants who sold WEPs in 7 traditional markets within 5 of the jurisdictions (Table 1, Fig. 1). We asked the same questions as those in the field interviews, including about WEPs that they regularly collect/sell even if they were not available at the time of the interview, and we also recorded their demographic variables. For the WEPs that were currently being sold at each stall, we recorded the prices of each.

We collected herbarium voucher specimens during field walks with participants, with initial identification being conducted on-site. Voucher specimens were later identified by J.W. and deposited in the Environmental Laboratory of Chengdu University (Table 2). Identification was carried out using keys, online plant databases, pictorial floras, plant dictionaries, and other taxonomic references, with accepted Latin names verified using The Plant List (www.theplantlist.org).

\section{Data analysis}

We grouped all WEPs into the following seven (non-exclusive) categories based on consumption pattern: primary food, famine food, snack, spice, culinary coagulant, medicine, and other uses. All data on participant demographics and the WEPs they identified were entered into a Microsoft Excel spreadsheet and organized for 
Table 2 The WEPs traditionally used by the Liangshan Yi

\begin{tabular}{|c|c|c|c|c|c|c|c|c|c|c|}
\hline Scientific name & Family name & $\begin{array}{l}\text { Growth } \\
\text { form }\end{array}$ & $\begin{array}{l}\text { Part used } \\
\text { (consumption pattern) }\end{array}$ & $\begin{array}{l}\text { Medicinal } \\
\text { uses(s) }\end{array}$ & $\begin{array}{l}\text { Collection } \\
\text { months }\end{array}$ & $\begin{array}{l}\text { Sold as/ } \\
\text { price }(¥ / \mathrm{kg})\end{array}$ & $\Sigma U s$ & UVs & $\begin{array}{l}\text { UV } \\
\text { rank }\end{array}$ & $\begin{array}{l}\text { Voucher } \\
\text { no. }\end{array}$ \\
\hline $\begin{array}{l}\text { Actinidia kolomikta } \\
\text { (Rupr. \& Maxim.) Maxim. }\end{array}$ & Actinidiaceae & Climber & Fruit (snack) & & $9-10$ & $\mathrm{~N}$ & 244 & 0.62 & 51 & LS0081 \\
\hline $\begin{array}{l}\text { Sambucus adnata Wall. } \\
\text { ex DC. }\end{array}$ & Adoxaceae & Herb & $\begin{array}{l}\text { Fruit (snack), } \\
\text { aboveground part } \\
\text { (medicine, other use) }\end{array}$ & $\begin{array}{l}\text { Bone fracture, } \\
\text { rheumatism }\end{array}$ & $1-12$ & N & 422 & 1.07 & 10 & LS0152 \\
\hline $\begin{array}{l}\text { Sambucus williamsii } \\
\text { Hance }\end{array}$ & Adoxaceae & Shrub & Leaf, bark (medicine) & Bone fracture & $1-12$ & N & 207 & 0.52 & 62 & LS0181 \\
\hline $\begin{array}{l}\text { Viburnum betulifolium } \\
\text { Batalin }\end{array}$ & Adoxaceae & Shrub & Fruit (snack, spice) & & $9-10$ & N & 249 & 0.63 & 49 & LS0125 \\
\hline Amaranthus blitum L. & Amaranthaceae & Herb & $\begin{array}{l}\text { Tender shoot } \\
\text { (primary food) }\end{array}$ & & $3-6$ & N & 233 & 0.59 & 54 & LS0166 \\
\hline Celosia argentea $\mathrm{L}$. & Amaranthaceae & Herb & Seed (famine food) & & $7-10$ & N & 99 & 0.25 & 99 & LS0093 \\
\hline Chenopodium hybridum L. & Amaranthaceae & Herb & $\begin{array}{l}\text { Tender shoot } \\
\text { (primary food) }\end{array}$ & & $4-6$ & N & 287 & 0.72 & 38 & LS0016 \\
\hline $\begin{array}{l}\text { Allium macrostemon } \\
\text { Bunge }\end{array}$ & Amaryllidaceae & Herb & $\begin{array}{l}\text { Whole plant (primary } \\
\text { food, medicine, spice) }\end{array}$ & Gastropathy & $3-11$ & N & 228 & 0.58 & 55 & LS0147 \\
\hline $\begin{array}{l}\text { Allium ovalifolium } \\
\text { Hand.-Mazz. }\end{array}$ & Amaryllidaceae & Herb & Leaf (primary food) & & $3-10$ & N & 131 & 0.33 & 90 & LS0177 \\
\hline $\begin{array}{l}\text { Toxicodendron } \\
\text { vernicifluum (Stokes) } \\
\text { F.A. Barkley }\end{array}$ & Anacardiaceae & Tree & $\begin{array}{l}\text { Tender shoot (primary } \\
\text { food), branch (other use) }\end{array}$ & & $3-5$ & N & 213 & 0.54 & 61 & LS0140 \\
\hline $\begin{array}{l}\text { Angelica sinensis } \\
\text { (Oliv.) Diels }\end{array}$ & Apiaceae & Herb & $\begin{array}{l}\text { Root (primary food, } \\
\text { medicine) }\end{array}$ & Tonify & $6-9$ & $\begin{array}{l}\text { Medicine/40 } \\
\text { (wet) }\end{array}$ & 371 & 0.94 & 15 & LS0043 \\
\hline $\begin{array}{l}\text { Oenanthe javanica } \\
\text { (Blume) DC. }\end{array}$ & Apiaceae & Herb & $\begin{array}{l}\text { Aboveground part } \\
\text { (primary food) }\end{array}$ & & $3-6$ & N & 258 & 0.65 & 45 & LS0038 \\
\hline $\begin{array}{l}\text { Metaplexis japonica } \\
\text { (Thunb.) Makino }\end{array}$ & Apocynaceae & Climber & Fruit (snack) & & $8-10$ & N & 130 & 0.33 & 91 & LS0032 \\
\hline Aralia chinensis L. & Araliaceae & Tree & $\begin{array}{l}\text { Tender shoot (primary } \\
\text { food), branch (other use) }\end{array}$ & & $3-5$ & Food/10 & 269 & 0.68 & 41 & LS0375 \\
\hline $\begin{array}{l}\text { Aristolochia versicolor } \\
\text { S.M.Hwang }\end{array}$ & Aristolochiaceae & Climber & Root (medicine) & $\begin{array}{l}\text { Headache, } \\
\text { injury, } \\
\text { gastroenteritis }\end{array}$ & $4-10$ & Medicine/200 & 52 & 0.13 & 105 & LS0193 \\
\hline $\begin{array}{l}\text { Polygonatum } \\
\text { cyrtonema Hua }\end{array}$ & Asparagaceae & Herb & $\begin{array}{l}\text { Root (primary food, } \\
\text { medicine) }\end{array}$ & Tonify & $5-8$ & Medicine/40 & 334 & 0.84 & 29 & LS0536 \\
\hline $\begin{array}{l}\text { Begonia grandis subsp. } \\
\text { sinensis (A.DC.) Irmsch. }\end{array}$ & Begoniaceae & Herb & Stem (primary food) & & $4-10$ & N & 140 & 0.35 & 85 & LS0151 \\
\hline $\begin{array}{l}\text { Berberis jamesiana } \\
\text { Forrest \& W.W.Sm. }\end{array}$ & Berberidaceae & Shrub & $\begin{array}{l}\text { Fruit (culinary coagulant, } \\
\text { snack, spice), root } \\
\text { (medicine) }\end{array}$ & Diarrhea & $9-12$ & N & 761 & 1.92 & 1 & LS0248 \\
\hline $\begin{array}{l}\text { Mahonia bealei } \\
\text { (Fortune) Pynaert }\end{array}$ & Berberidaceae & Shrub & $\begin{array}{l}\text { Fruit (snack), bark and } \\
\text { root (medicine) }\end{array}$ & Diarrhea & $1-12$ & Medicine/50 & 204 & 0.52 & 65 & LS0062 \\
\hline Incarvillea diffusa Royle & Bignoniaceae & Herb & $\begin{array}{l}\text { Aboveground part } \\
\text { (medicine) }\end{array}$ & Hepatitis & $1-12$ & $\mathrm{~N}$ & 214 & 0.54 & 60 & LS0558 \\
\hline $\begin{array}{l}\text { Cynoglossum amabile } \\
\text { Stapf \& J.R.Drumm. }\end{array}$ & Boraginaceae & Herb & Root (medicine) & $\begin{array}{l}\text { Hemorrhoid, } \\
\text { enteritis }\end{array}$ & $3-10$ & $\mathrm{~N}$ & 198 & 0.50 & 68 & LS0013 \\
\hline $\begin{array}{l}\text { Capsella bursa-pastoris } \\
\text { (L.) Medik. }\end{array}$ & Brassicaceae & Herb & $\begin{array}{l}\text { Tender shoot } \\
\text { (primary food) }\end{array}$ & & $3-5$ & $\mathrm{~N}$ & 155 & 0.39 & 84 & LS0423 \\
\hline $\begin{array}{l}\text { Cardamine tangutorum } \\
\text { O.E.Schulz }\end{array}$ & Brassicaceae & Herb & $\begin{array}{l}\text { Tender shoot (primary } \\
\text { food, medicine) }\end{array}$ & Hypertension & $3-5$ & Food/10 & 273 & 0.69 & 39 & LS0279 \\
\hline $\begin{array}{l}\text { Nasturtium officinale } \\
\text { R.Br. }\end{array}$ & Brassicaceae & Herb & $\begin{array}{l}\text { Aboveground part } \\
\text { (primary food) }\end{array}$ & & $4-10$ & Food/5 & 139 & 0.35 & 87 & LS0063 \\
\hline $\begin{array}{l}\text { Rorippa dubia } \\
\text { (Pers.) H.Hara }\end{array}$ & Brassicaceae & Herb & $\begin{array}{l}\text { Tender shoot } \\
\text { (primary food) }\end{array}$ & & $3-6$ & $\mathrm{~N}$ & 107 & 0.27 & 95 & LS0121 \\
\hline $\begin{array}{l}\text { Hylocereus undatus } \\
\text { (Haw.) Britton \& Rose }\end{array}$ & Cactaceae & Shrub & Flower (primary food) & & $7-11$ & $\mathrm{~N}$ & 98 & 0.25 & 100 & LS0162 \\
\hline Opuntia ficus-indica & Cactaceae & Shrub & Stem (primary food, & Tonsillitis & $1-12$ & N & 294 & 0.74 & 37 & LS0346 \\
\hline
\end{tabular}


Table 2 The WEPs traditionally used by the Liangshan Yi (Continued)

\begin{tabular}{|c|c|c|c|c|c|c|c|c|c|c|}
\hline Scientific name & Family name & $\begin{array}{l}\text { Growth } \\
\text { form }\end{array}$ & $\begin{array}{l}\text { Part used } \\
\text { (consumption pattern) }\end{array}$ & $\begin{array}{l}\text { Medicinal } \\
\text { uses(s) }\end{array}$ & $\begin{array}{l}\text { Collection } \\
\text { months }\end{array}$ & $\begin{array}{l}\text { Sold as/ } \\
\text { price }(¥ / \mathrm{kg})\end{array}$ & $\Sigma U s$ & UVs & $\begin{array}{l}\text { UV } \\
\text { rank }\end{array}$ & $\begin{array}{l}\text { Voucher } \\
\text { no. }\end{array}$ \\
\hline (L.) Mill. & & & medicine), fruit (snack) & & & & & & & \\
\hline $\begin{array}{l}\text { Codonopsis pilosula } \\
\text { subsp. tangshen (Oliv.) } \\
\text { D.Y.Hong }\end{array}$ & Campanulaceae & Herb & $\begin{array}{l}\text { Root (primary food, } \\
\text { medicine) }\end{array}$ & $\begin{array}{l}\text { Tonify, } \\
\text { gallstone }\end{array}$ & $4-8$ & Medicine/50 & 342 & 0.86 & 27 & LS0223 \\
\hline $\begin{array}{l}\text { Leycesteria formosa } \\
\text { Wall. }\end{array}$ & Caprifoliaceae & Shrub & Tender shoot (medicine) & Measles & $3-10$ & N & 132 & 0.33 & 89 & LS0509 \\
\hline Arctium lappa $\mathrm{L}$. & Compositae & Herb & $\begin{array}{l}\text { Root (primary food, } \\
\text { medicine) }\end{array}$ & $\begin{array}{l}\text { Tonify, } \\
\text { detoxify }\end{array}$ & $3-5$ & Medicine/20 & 382 & 0.96 & 14 & LS0315 \\
\hline $\begin{array}{l}\text { Artemisia capillaris } \\
\text { Thunb. }\end{array}$ & Compositae & Herb & $\begin{array}{l}\text { Tender shoot (famine } \\
\text { food), aboveground part } \\
\text { (medicine, other use) }\end{array}$ & Injury & $3-10$ & N & 569 & 1.46 & 3 & LS0256 \\
\hline Cirsium shansiense Petr. & Compositae & Herb & $\begin{array}{l}\text { Root (primary food, } \\
\text { medicine) }\end{array}$ & $\begin{array}{l}\text { Tonify, } \\
\text { nephrosis }\end{array}$ & $3-11$ & Medicine/12 & 452 & 1.14 & 7 & LS0362 \\
\hline Eclipta prostrata (L.) L. & Compositae & Herb & Whole plant (medicine) & $\begin{array}{l}\text { Diarrhea, } \\
\text { cough, } \\
\text { pneumonia }\end{array}$ & $3-10$ & N & 168 & 0.42 & 79 & LS0033 \\
\hline $\begin{array}{l}\text { Kalimeris indica (L.) } \\
\text { Sch.Bip. }\end{array}$ & Compositae & Herb & $\begin{array}{l}\text { Tender shoot (primary } \\
\text { food), root (medicine) }\end{array}$ & Diarrhea & $3-10$ & N & 140 & 0.35 & 86 & LSO255 \\
\hline $\begin{array}{l}\text { Pseudognaphalium } \\
\text { affine (D.Don) Anderb. }\end{array}$ & Compositae & Herb & $\begin{array}{l}\text { Flower (famine food), } \\
\text { whole plant (other use) }\end{array}$ & & $2-5$ & N & 190 & 0.48 & 71 & LS0014 \\
\hline Sonchus oleraceus (L.) L. & Compositae & Herb & $\begin{array}{l}\text { Tender shoot } \\
\text { (primary food) }\end{array}$ & & $3-6$ & N & 201 & 0.51 & 67 & LS0463 \\
\hline $\begin{array}{l}\text { Taraxacum mongolicum } \\
\text { Hand.-Mazz. }\end{array}$ & Compositae & Herb & $\begin{array}{l}\text { Leaf (primary food), } \\
\text { whole plant (medicine) }\end{array}$ & Cough & $3-10$ & Medicine/5 & 334 & 0.84 & 30 & LS0410 \\
\hline $\begin{array}{l}\text { Cornus kousa subsp. } \\
\text { chinensis (Osborn) } \\
\text { Q.Y.Xiang }\end{array}$ & Cornaceae & Tree & Fruit (snack) & & $9-10$ & Snack/10 & 196 & 0.49 & 70 & LS0407 \\
\hline $\begin{array}{l}\text { Trichosanthes kirilowii } \\
\text { Maxim. }\end{array}$ & Cucurbitaceae & Climber & Flower (medicine) & Cough & $5-8$ & N & 114 & 0.29 & 94 & LS0058 \\
\hline $\begin{array}{l}\text { Araiostegia divaricata } \\
\text { var. formosana (Hayata) } \\
\text { M. Kato }\end{array}$ & Davalliaceae & Herb & Root (medicine) & Hypertension & $3-10$ & N & 67 & 0.17 & 104 & LS0579 \\
\hline $\begin{array}{l}\text { Pteridium aquilinum } \\
\text { (L.) Kuhn }\end{array}$ & Dennstaedtiaceae & Herb & $\begin{array}{l}\text { Tender shoot (primary } \\
\text { food), root (famine food) }\end{array}$ & & $3-6$ & Food/10 & 536 & 1.35 & 4 & LS0004 \\
\hline $\begin{array}{l}\text { Dioscorea polystachya } \\
\text { Turcz. }\end{array}$ & Dioscoreaceae & Climber & $\begin{array}{l}\text { Root (primary food), } \\
\text { bulbil (snack) }\end{array}$ & & $3-10$ & N & 329 & 0.83 & 33 & LS0376 \\
\hline Diospyros lotus L. & Ebenaceae & Tree & Fruit (snack) & & $10-11$ & N & 170 & 0.43 & 78 & LS0467 \\
\hline $\begin{array}{l}\text { Elaeagnus pungens } \\
\text { Thunb. }\end{array}$ & Elaeagnaceae & Shrub & Fruit (snack) & & $8-9$ & N & 346 & 0.87 & 23 & LS0221 \\
\hline Equisetum giganteum L. & Equisetaceae & Herb & Whole plant (medicine) & $\begin{array}{l}\text { Cold, } \\
\text { headache, } \\
\text { stomachache }\end{array}$ & $3-10$ & N & 102 & 0.26 & 97 & LS0303 \\
\hline $\begin{array}{l}\text { Vaccinium fragile } \\
\text { Franch. }\end{array}$ & Ericaceae & Shrub & Fruit (snack) & & $7-10$ & N & 224 & 0.57 & 57 & LS0452 \\
\hline $\begin{array}{l}\text { Eucommia ulmoides } \\
\text { Oliv. }\end{array}$ & Eucommiaceae & Tree & $\begin{array}{l}\text { Bark (primary food, } \\
\text { medicine) }\end{array}$ & Nephropathy & $1-12$ & N & 178 & 0.45 & 76 & LS0199 \\
\hline $\begin{array}{l}\text { Quercus schottkyana } \\
\text { Rehder \& E.H.Wilson }\end{array}$ & Fagaceae & Tree & Seed (snack) & & $10-11$ & N & 156 & 0.39 & 83 & LS0366 \\
\hline $\begin{array}{l}\text { Helwingia japonica } \\
\text { (Thunb.) F.Dietr. }\end{array}$ & Helwingiaceae & Shrub & Leaf (primary food) & & $3-6$ & N & 105 & 0.27 & 96 & LS0319 \\
\hline Iris forrestii Dykes & Iridaceae & Herb & Root (medicine) & Cough & $3-10$ & N & 178 & 0.45 & 77 & LS0417 \\
\hline Mentha canadensis L. & Lamiaceae & Herb & $\begin{array}{l}\text { Tender shoot (primary } \\
\text { food, medicine, spice) }\end{array}$ & Hyperthermia & $3-10$ & Spice/10 & 393 & 0.99 & 12 & LS0403 \\
\hline $\begin{array}{l}\text { Perilla frutescens } \\
\text { (L.) Britton }\end{array}$ & Lamiaceae & Herb & $\begin{array}{l}\text { Tender shoot (primary } \\
\text { food), seed (Spice) }\end{array}$ & & $8-10$ & $\mathrm{~N}$ & 438 & 1.11 & 8 & LS0292 \\
\hline Akebia trifoliata & Lardizabalaceae & Climber & Fruit (snack) & & $7-8$ & N & 236 & 0.60 & 52 & LS0481 \\
\hline
\end{tabular}


Table 2 The WEPs traditionally used by the Liangshan Yi (Continued)

\begin{tabular}{|c|c|c|c|c|c|c|c|c|c|c|}
\hline Scientific name & Family name & $\begin{array}{l}\text { Growth } \\
\text { form }\end{array}$ & $\begin{array}{l}\text { Part used } \\
\text { (consumption pattern) }\end{array}$ & $\begin{array}{l}\text { Medicinal } \\
\text { uses(s) }\end{array}$ & $\begin{array}{l}\text { Collection } \\
\text { months }\end{array}$ & $\begin{array}{l}\text { Sold as/ } \\
\text { price }(¥ / \mathrm{kg})\end{array}$ & $\Sigma$ Us & UVs & $\begin{array}{l}\text { UV } \\
\text { rank }\end{array}$ & $\begin{array}{l}\text { Voucher } \\
\text { no. }\end{array}$ \\
\hline \multicolumn{11}{|l|}{ (Thunb.) Koidz. } \\
\hline $\begin{array}{l}\text { Decaisnea insignis } \\
\text { (Griff.) Hook.f. \& } \\
\text { Thomson }\end{array}$ & Lardizabalaceae & Shrub & Fruit (snack) & & $10-11$ & N & 167 & 0.42 & 80 & LS0200 \\
\hline $\begin{array}{l}\text { Litsea cubeba } \\
\text { (Lour.) Pers. }\end{array}$ & Lauraceae & Tree & Fruit and root (spice) & & $7-10$ & Spice/40 & 205 & 0.52 & 64 & LS0561 \\
\hline Litsea pungens Hemsl. & Lauraceae & Tree & Fruit and root (spice) & & $7-10$ & Spice/40 & 343 & 0.87 & 25 & LS0535 \\
\hline $\begin{array}{l}\text { Pueraria montana var. } \\
\text { lobata (Willd.) Sanjappa } \\
\text { \& Pradeep }\end{array}$ & Leguminosae & Climber & Root (snack) & & $7-10$ & Snack/20 & 183 & 0.46 & 74 & LS0448 \\
\hline $\begin{array}{l}\text { Spatholobus } \\
\text { suberectus Dunn }\end{array}$ & Leguminosae & Climber & Stem (medicine) & Heart disease & $3-10$ & Medicine/40 & 89 & 0.22 & 102 & LS0457 \\
\hline Vicia sativa $\mathrm{L}$. & Leguminosae & Herb & $\begin{array}{l}\text { Tender shoot } \\
\text { (primary food) }\end{array}$ & & $1-12$ & $\mathrm{~N}$ & 189 & 0.48 & 72 & LS0252 \\
\hline $\begin{array}{l}\text { Fritillaria cirrhosa } \\
\text { D.Don }\end{array}$ & Liliaceae & Herb & Bulb (medicine) & Cough, injury & $7-8$ & $\begin{array}{l}\text { Medicine/ } \\
\text { 2000(dry) }\end{array}$ & 261 & 0.66 & 44 & LS0471 \\
\hline $\begin{array}{l}\text { Huperzia squarrosa } \\
\text { (G. Forst.) Trevis. }\end{array}$ & Lycopodiaceae & Herb & Whole plant (medicine) & $\begin{array}{l}\text { Rheumatism, } \\
\text { gastropathy }\end{array}$ & $3-10$ & Medicine/50 & 78 & 0.20 & 103 & LS0532 \\
\hline $\begin{array}{l}\text { Lycopodium japonicum } \\
\text { Thunb. }\end{array}$ & Lycopodiaceae & Herb & Spore powder (medicine) & Rheumatism & $3-10$ & Medicine/200 & 95 & 0.24 & 101 & LS0441 \\
\hline Malva verticillata $\mathrm{L}$. & Malvaceae & Herb & Whole plant (medicine) & Delivery & $1-12$ & N & 270 & 0.68 & 40 & LS0022 \\
\hline Paris polyphylla Sm. & Melanthiaceae & Herb & Root (medicine) & $\begin{array}{l}\text { Muscle pain, } \\
\text { injury }\end{array}$ & $3-10$ & Medicine/600 & 254 & 0.64 & 46 & LS0424 \\
\hline $\begin{array}{l}\text { Toona sinensis } \\
\text { (Juss.) M.Roem. }\end{array}$ & Meliaceae & Tree & $\begin{array}{l}\text { Tender shoot (primary } \\
\text { food, medicine) }\end{array}$ & Diarrhea & $3-5$ & N & 354 & 0.89 & 21 & LS0369 \\
\hline Ficus pumila L. & Moraceae & Climber & Fruit (snack) & & $6-8$ & N & 115 & 0.29 & 93 & LS0161 \\
\hline Ficus tikoua Bureau & Moraceae & Shrub & Fruit (snack) & & $7-8$ & N & 295 & 0.74 & 36 & LS0148 \\
\hline Morus australis Poir. & Moraceae & Tree & Fruit (snack) & & $4-5$ & N & 253 & 0.64 & 47 & LS0389 \\
\hline $\begin{array}{l}\text { Musa basjoo Siebold \& } \\
\text { Zucc. ex linuma }\end{array}$ & Musaceae & Tree & $\begin{array}{l}\text { Flower (medicine), } \\
\text { fruit(snack) }\end{array}$ & Heart disease & $1-12$ & N & 207 & 0.52 & 63 & LS0098 \\
\hline Myrica nana A. Chev. & Myricaceae & Shrub & Fruit (snack) & & $6-8$ & Snack/10 & 198 & 0.50 & 69 & LS0225 \\
\hline $\begin{array}{l}\text { Matteuccia struthiopteris } \\
\text { (L.) Tod. }\end{array}$ & Onocleaceae & Herb & $\begin{array}{l}\text { Tender shoot } \\
\text { (primary food) }\end{array}$ & & $3-5$ & Food/10 & 345 & 0.87 & 24 & LS0272 \\
\hline $\begin{array}{l}\text { Ophioglossum } \\
\text { vulgatum L. }\end{array}$ & Ophioglossaceae & Herb & $\begin{array}{l}\text { Whole plant (primary } \\
\text { food, medicine) }\end{array}$ & Tonify & $3-10$ & Medicine/200 & 321 & 0.81 & 34 & LS0446 \\
\hline $\begin{array}{l}\text { Bulbophyllum } \\
\text { odoratissimum (Sm.) } \\
\text { Lindl. ex Wall. }\end{array}$ & Orchidaceae & Herb & Whole plant (medicine) & Cough & $7-10$ & Medicine/40 & 100 & 0.25 & 98 & LS0569 \\
\hline Gastrodia elata & Orchidaceae & Herb & $\begin{array}{l}\text { Rhizome } \\
\text { (primary food, medicine) }\end{array}$ & Headache & $3-6$ & $\begin{array}{l}\text { Medicine/100 } \\
\text { (wet), } 500 \text { (dry) }\end{array}$ & 430 & 1.09 & 9 & LS0142 \\
\hline $\begin{array}{l}\text { Osmunda japonica } \\
\text { Thunb. }\end{array}$ & Osmundaceae & Herb & $\begin{array}{l}\text { Tender shoot } \\
\text { (primary food) }\end{array}$ & & $3-5$ & N & 332 & 0.84 & 32 & LS0367 \\
\hline Oxalis corniculata $\mathrm{L}$. & Oxalidaceae & Herb & $\begin{array}{l}\text { Aboveground part (snack, } \\
\text { spice, other use) }\end{array}$ & & $1-12$ & N & 461 & 1.16 & 6 & LS0343 \\
\hline Plantago major $\mathrm{L}$. & Plantaginaceae & Herb & $\begin{array}{l}\text { Aboveground part } \\
\text { (primary food, medicine) }\end{array}$ & $\begin{array}{l}\text { Diarrhea, } \\
\text { cough }\end{array}$ & $3-10$ & Medicine/5 & 416 & 1.05 & 11 & LS0007 \\
\hline Fargesia spathacea Franch. & Poaceae & Shrub & $\begin{array}{l}\text { Tender shoot (primary } \\
\text { food) }\end{array}$ & & $3-5$ & Food/14 & 357 & 0.90 & 17 & LS0087 \\
\hline $\begin{array}{l}\text { Imperata cylindrica (L.) } \\
\text { Raeusch. }\end{array}$ & Poaceae & Herb & $\begin{array}{l}\text { Root (primary food, } \\
\text { medicine) }\end{array}$ & $\begin{array}{l}\text { Nosebleed, } \\
\text { cough }\end{array}$ & $3-10$ & N & 295 & 0.74 & 35 & LS0259 \\
\hline $\begin{array}{l}\text { Reynoutria multiflora } \\
\text { (Thunb.) Moldenke }\end{array}$ & Polygonaceae & Herb & $\begin{array}{l}\text { Root (medicine), leaf } \\
\text { (culinary coagulant) }\end{array}$ & Headache & $1-12$ & Medicine/40 & 222 & 0.56 & 58 & LS0549 \\
\hline $\begin{array}{l}\text { Lemmaphyllum carnosum } \\
\text { (J. Sm. ex Hook.) C. Presl }\end{array}$ & Polypodiaceae & Herb & $\begin{array}{l}\text { Aboveground part } \\
\text { (medicine) }\end{array}$ & Cough, injury & $3-10$ & $\mathrm{~N}$ & 163 & 0.41 & 81 & LS0349 \\
\hline
\end{tabular}


Table 2 The WEPs traditionally used by the Liangshan Yi (Continued)

\begin{tabular}{|c|c|c|c|c|c|c|c|c|c|c|}
\hline Scientific name & Family name & $\begin{array}{l}\text { Growth } \\
\text { form }\end{array}$ & $\begin{array}{l}\text { Part used } \\
\text { (consumption pattern) }\end{array}$ & $\begin{array}{l}\text { Medicinal } \\
\text { uses(s) }\end{array}$ & $\begin{array}{l}\text { Collection } \\
\text { months }\end{array}$ & $\begin{array}{l}\text { Sold as/ } \\
\text { price ( } \neq / \mathrm{kg})\end{array}$ & $\Sigma$ Us & UVs & $\begin{array}{l}\text { UV } \\
\text { rank }\end{array}$ & $\begin{array}{l}\text { Voucher } \\
\text { no. }\end{array}$ \\
\hline $\begin{array}{l}\text { Pyrrosia lingua (Thunb.) } \\
\text { Farw. }\end{array}$ & Polypodiaceae & Herb & Whole plant (medicine) & Gallstone & $3-10$ & $\mathrm{~N}$ & 138 & 0.35 & 88 & LS0141 \\
\hline $\begin{array}{l}\text { Lysimachia congestiflora } \\
\text { Hemsl. }\end{array}$ & Primulaceae & Herb & Whole plant (medicine) & Gallstone & $3-10$ & N & 220 & 0.56 & 59 & LS0172 \\
\hline $\begin{array}{l}\text { Anemone vitifolia Buch.- } \\
\text { Ham. ex DC. }\end{array}$ & Ranunculaceae & Herb & Fruit (famine food) & & $9-12$ & N & 263 & 0.66 & 43 & LS0328 \\
\hline Hovenia dulcis Thunb. & Rhamnaceae & Tree & $\begin{array}{l}\text { Infructescence shaft } \\
\text { (snack, medicine) }\end{array}$ & Tonify & $8-10$ & Snack/14 & 204 & 0.52 & 66 & LS0300 \\
\hline Agrimonia pilosa Ledeb. & Rosaceae & Herb & $\begin{array}{l}\text { Aboveground part } \\
\text { (medicine) }\end{array}$ & Diarrhea & $1-12$ & N & 179 & 0.45 & 75 & LS0224 \\
\hline $\begin{array}{l}\text { Crataegus scabrifolia } \\
\text { (Franch.) Rehder }\end{array}$ & Rosaceae & Tree & Fruit (snack, medicine) & Cough & $8-10$ & $\mathrm{~N}$ & 354 & 0.89 & 20 & LS0179 \\
\hline $\begin{array}{l}\text { Duchesnea indica (Jacks.) } \\
\text { Focke }\end{array}$ & Rosaceae & Herb & Fruit (snack) & & $6-10$ & N & 363 & 0.92 & 16 & LS0427 \\
\hline $\begin{array}{l}\text { Fragaria nilgerrensis Schltdl. } \\
\text { ex J.Gay }\end{array}$ & Rosaceae & Herb & Fruit (snack) & & $5-9$ & N & 356 & 0.90 & 18 & LS0124 \\
\hline Potentilla discolor Bunge & Rosaceae & Herb & Whole plant (medicine) & $\begin{array}{l}\text { Diarrhea, } \\
\text { gastropathy }\end{array}$ & $3-10$ & N & 245 & 0.62 & 50 & LS0385 \\
\hline Prunus trichostoma Koehne & Rosaceae & Tree & Fruit (snack) & & $7-10$ & N & 225 & 0.57 & 56 & LS0180 \\
\hline $\begin{array}{l}\text { Pyracantha fortuneana } \\
\text { (Maxim.) H.L.Li }\end{array}$ & Rosaceae & Shrub & $\begin{array}{l}\text { Fruit (famine food, snack, } \\
\text { other use) }\end{array}$ & & $1-12$ & N & 741 & 1.87 & 2 & LS0404 \\
\hline $\begin{array}{l}\text { Pyrus pashia Buch.-Ham. ex } \\
\text { D.Don }\end{array}$ & Rosaceae & Tree & Fruit (snack) & & $9-10$ & N & 340 & 0.86 & 28 & LS0483 \\
\hline Rosa omeiensis Rolfe & Rosaceae & Herb & Fruit (snack) & & $5-8$ & Snack/10 & 385 & 0.97 & 13 & LS0337 \\
\hline Rosa roxburghii Tratt. & Rosaceae & Shrub & Fruit (snack) & & $8-10$ & Snack/10 & 236 & 0.60 & 53 & LS0021 \\
\hline $\begin{array}{l}\text { Rubus ellipticus var. } \\
\text { obcordatus (Franch.) Focke }\end{array}$ & Rosaceae & Shrub & Fruit (snack) & & $4-5$ & N & 333 & 0.84 & 31 & LS0055 \\
\hline $\begin{array}{l}\text { Rubus inopertus (Focke) } \\
\text { Focke }\end{array}$ & Rosaceae & Shrub & Fruit (snack) & & $7-8$ & N & 343 & 0.87 & 26 & LS0023 \\
\hline Rubus mesogaeus Focke & Rosaceae & Shrub & Fruit (snack) & & $7-8$ & N & 348 & 0.88 & 22 & LS0566 \\
\hline $\begin{array}{l}\text { Rubus wallichianus Wight } \\
\text { \& Arn. }\end{array}$ & Rosaceae & Shrub & Fruit (snack) & & $5-6$ & N & 356 & 0.90 & 19 & LS0035 \\
\hline Houttuynia cordata Thunb. & Saururaceae & Herb & $\begin{array}{l}\text { Whole plant (primary food, } \\
\text { medicine), root (spice) }\end{array}$ & Dyspepsia & $3-10$ & Food/6 & 500 & 1.26 & 5 & LS0056 \\
\hline $\begin{array}{l}\text { Schisandra rubriflora } \\
\text { Rehder \& E.H.Wilson }\end{array}$ & Schisandraceae & Climber & Fruit (snack, medicine) & Tonify & $9-12$ & N & 264 & 0.67 & 42 & LS0123 \\
\hline Smilax stans Maxim. & Smilacaceae & Shrub & $\begin{array}{l}\text { Tender hoot } \\
\text { (primary food) }\end{array}$ & & $4-8$ & Food/16 & 162 & 0.41 & 82 & LS0127 \\
\hline Physalis alkekengi L. & Solanaceae & Herb & Fruit (snack) & & $6-10$ & N & 128 & 0.32 & 92 & LS0130 \\
\hline $\begin{array}{l}\text { Vitis heyneana Roem. \& } \\
\text { Schult. }\end{array}$ & Vitaceae & Climber & Fruit (snack) & & $6-10$ & N & 187 & 0.47 & 73 & LS0017 \\
\hline Hemerocallis citrina Baroni & Xanthorrhoeaceae & Herb & Flower (primary food) & & $5-8$ & N & 251 & 0.63 & 48 & LS0500 \\
\hline
\end{tabular}

statistical analysis. Following the methods of Regassa et al. [20], we calculated the descriptive statistics on the number and percentage of species, genera, and families of WEPs, as well as their growth forms and the parts consumed. We calculated the use values for each species of WEP. The formula we used was adapted from Phillips and Gentry [52] by first considering a single participant interview [11]:

$$
\mathrm{UV}_{s}=\Sigma \mathrm{U}_{s} / n
$$

Where $\mathrm{UV}_{s}$ refers to the use value of a particular species "s," $n$ is the total number of respondents in the sample $(n=396)$, and $U_{s}$ refers to the number of citations of use mentioned by each participant for a particular species "s." The use values for each species were compiled into a table for interpretation [11]. 


\section{Results}

\section{Taxonomic diversity of WEPs}

The interview participants reported 105 WEPs from 97 genera and 62 families (Table 2). The families with the largest representation were Rosaceae (14 species), followed by Compositae ( 8 species), Brassicaceae (4 species), and Adoxaceae, Amaranthaceae, Leguminosae, and Moraceae (3 species each). Amaryllidaceae, Apiaceae, Berberidaceae, Cactaceae, Lamiaceae, Lardizabalaceae, Lauraceae, Lycopodiaceae, Orchidaceae, Poaceae, and Polypodiaceae each had 2 species. The remaining 44 families were represented by a single species each. The majority of WEPs were herbs (58 species), followed by shrubs (21 species), trees (15 species), and climbing plants (11 species).

Due to the dialectal diversity in Liangshan and across our site locations, while documenting the WEPs utilized by the Liangshan Yi, we also documented multiple local names for certain species (Table 3).

\section{The preparation and consumption pattern of WEPs Primary foods}

Of the 7 primary consumption patterns of WEPs in Liangshan, 40 species are used as primary foods, and of these, the most commonly consumed parts are tender shoots (18 species) and roots (8 species). Usually, the tender shoots, leaves, and flowers are made into soups (5 species), pickled (5 species), eaten raw as salad greens (2 species), or eaten after boiling in water (21 species) (Table 4). The roots tend to be stewed with pork or chicken (11 species), not only for nourishment, but also for the prevention and treatment of diseases. For example, the roots of Arctium lappa, Cirsium shansiense, Codonopsis pilosula subsp. tangshen, and Ophioglossum vulgatum are generally stewed with chicken or pork used as a tonic. Gastrodia elata is stewed to relieve headaches, Arctium lappa is also stewed for detoxification, and Imperata cylindrical is stewed to reduce cough.

One of the distinctive aspects of the traditional Liangshan Yi cuisine is sour soups, with a bowl of sour soup at almost every meal (Fig. 2). There are five species of WEPs used for making pickled (lacto-fermented) vegetables that are the basis for these soups: Begonia grandis subsp. sinensis, Nasturtium officinale, Oenanthe javanica, Rorippa dubia, and Smilax stans. These are prepared by putting the plant materials in boiling water for 1-2 min, then placing them into a bucket, with the addition of some salt, sealed and left for about half a month. The fermented WEPs become sour in flavor, and they are then stewed with potatoes, beans, and chicken, or made alone into soup. Some of the most famous Yi dishes are suāncài tāng (pickle soup) and suāncài jī tāng (pickle and chicken soup).

\section{Famine foods}

Five species of WEPs are eaten as food supplements in times of famine, including the seeds with pappus of Anemone vitifolia, tender shoots of Artemisia capillaris, seeds of Celosia argentea, flowers of Pseudognaphalium affine, and the ground-up fruits of Pyracantha fortuneana. Each of these can be mixed with buckwheat flour or cornmeal, which increases the volume of cakes for meals and increases their nutritive value as well. A single WEP is used to extract starch during famines. The roots of Pteridium aquilinum are crushed in water, and the starch is obtained through sedimentation and filtration. This starch is then used for making cakes after it is dried.

\section{Snacks}

Most of the WEPs eaten as snacks (38 species) are wild fruits, such as Akebia trifoliata, Cornus kousa subsp. chinensis, Elaeagnus pungens, Fragaria nilgerrensis, Pyracantha fortuneana, and Rubus sp. These are often consumed by Yi children when they are herding livestock. The Yi shepherd children also roast bulbils of Dioscorea polystachya and the seeds of Quercus schottkyana for snacks in the wild. The fresh roots of Pueraria montana var. lobata, which are also sold in the market for about $¥ 20$ per kilogram, are cut into thin slices and eaten as snacks.

\section{Spices}

There are nine species of WEPs used as spices, among which six species are seasonings. These include Litsea pungens and L. cubeba widely used in the local cuisine, being essential seasonings in such distinctive Yi dishes as tuótuó ròu (lump pork), tuó tuó jī (lump chicken), and suāncài tāng (pickle soup; Fig. 2). They are prepared by crushing the fresh ripe fruits of $L$. pungens and $L$. cubeba or by scraping the roots with a knife to form a powder. Similarly, wild onion (Allium macrostemon) and mint (Mentha canadensis) can be added to beef and/or mutton soup to enhance their flavor profiles by masking the strong meaty taste. The seeds of Perilla frutescens are fried then ground into powder and mixed with the flour of Fagopyrum tataricum to season cakes. The chopped roots of Houttuynia cordata mixed with soy sauce, vinegar, salt, and chili powder are used as a seasoning sauce (Fig. 3). The Liangshan Yi utilize three WEPs as flavor enhancers to increase the sourness of the soups beyond what the pickles provide, including the fruits of Berberis jamesiana and Viburnum betulifolium, as well as the leaves of Oxalis corniculata.

\section{Culinary coagulants}

There are two species used as culinary coagulants. The leaves of Reynoutria multiflora are crushed and put in 


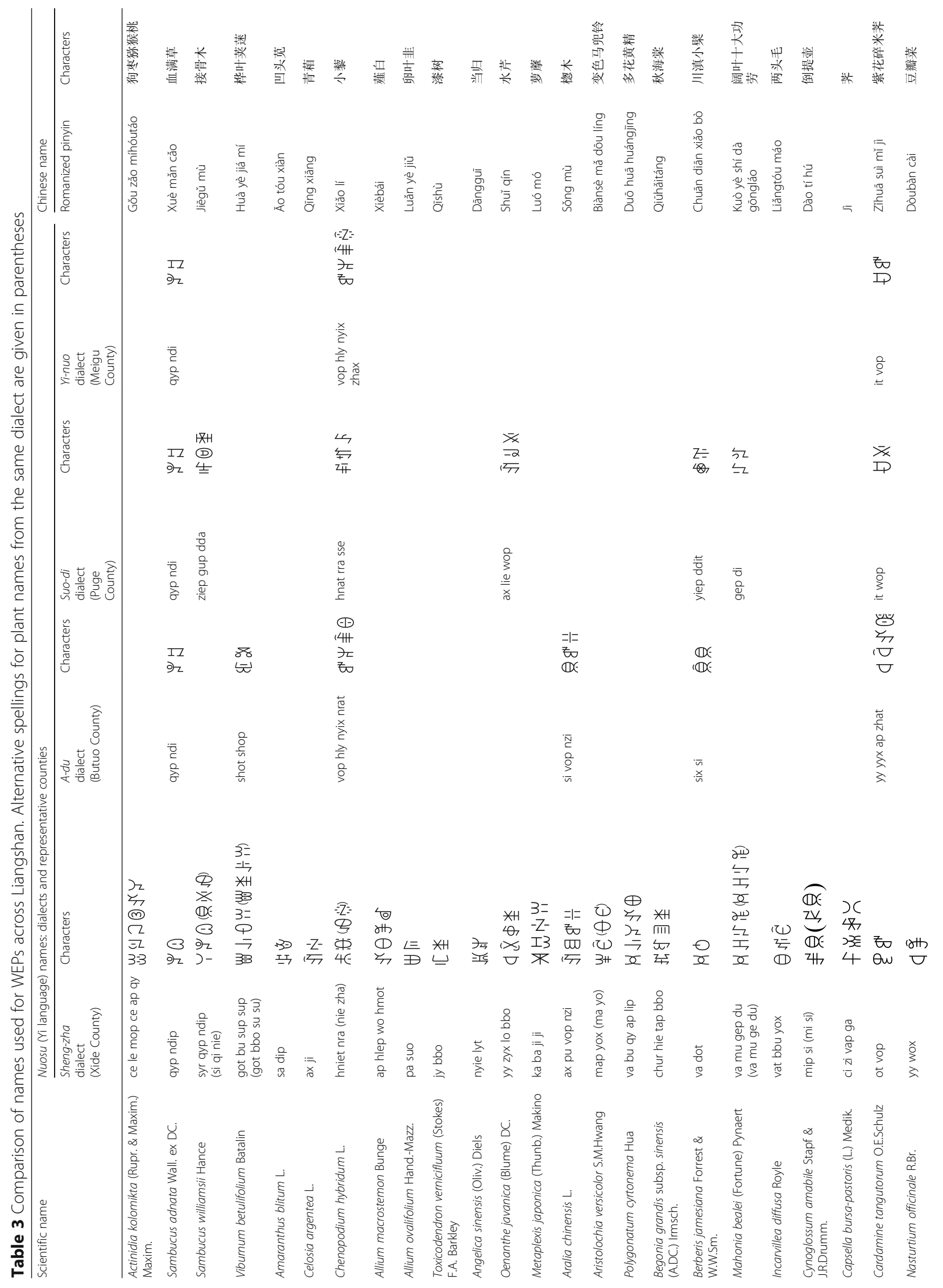




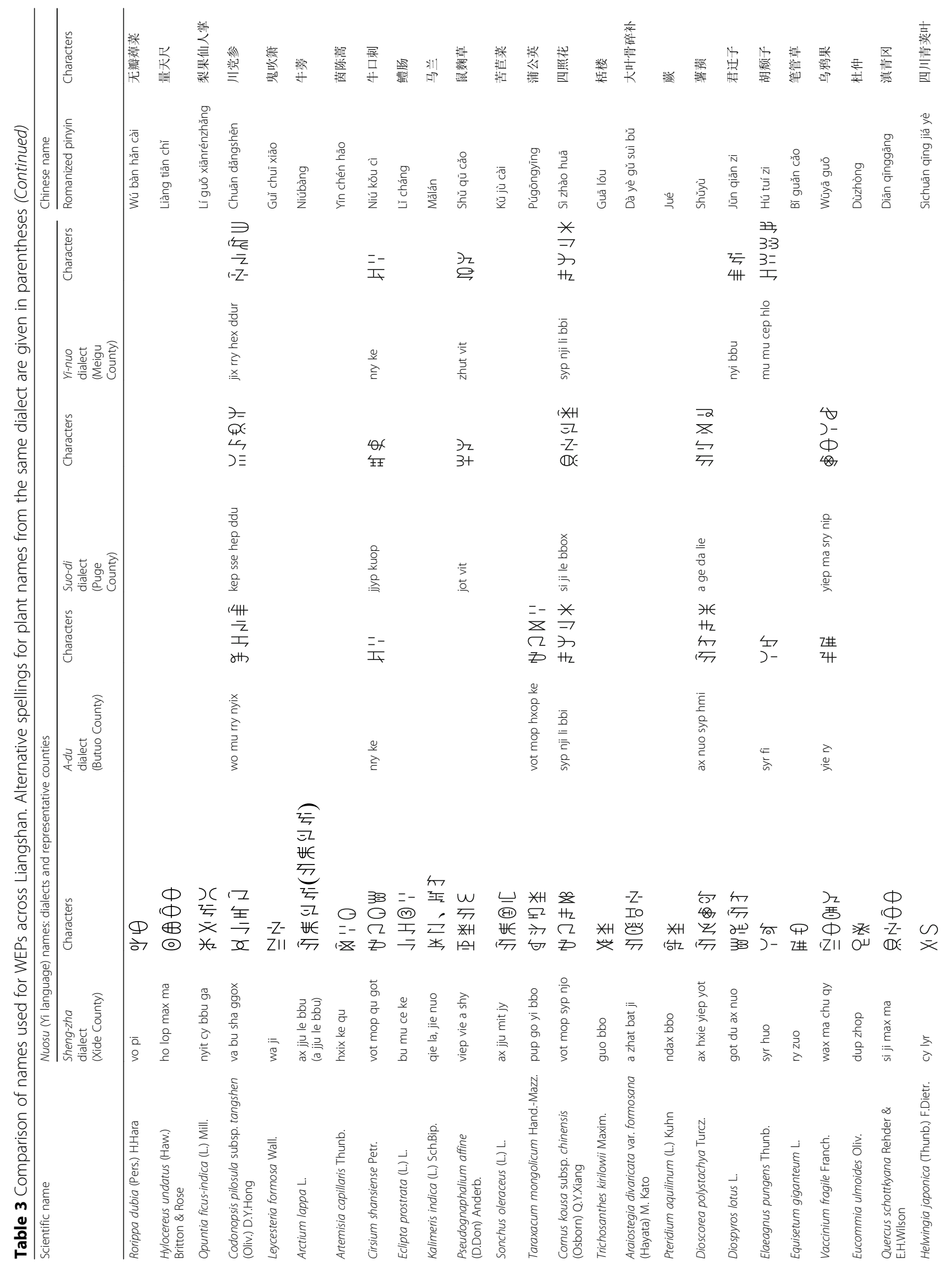




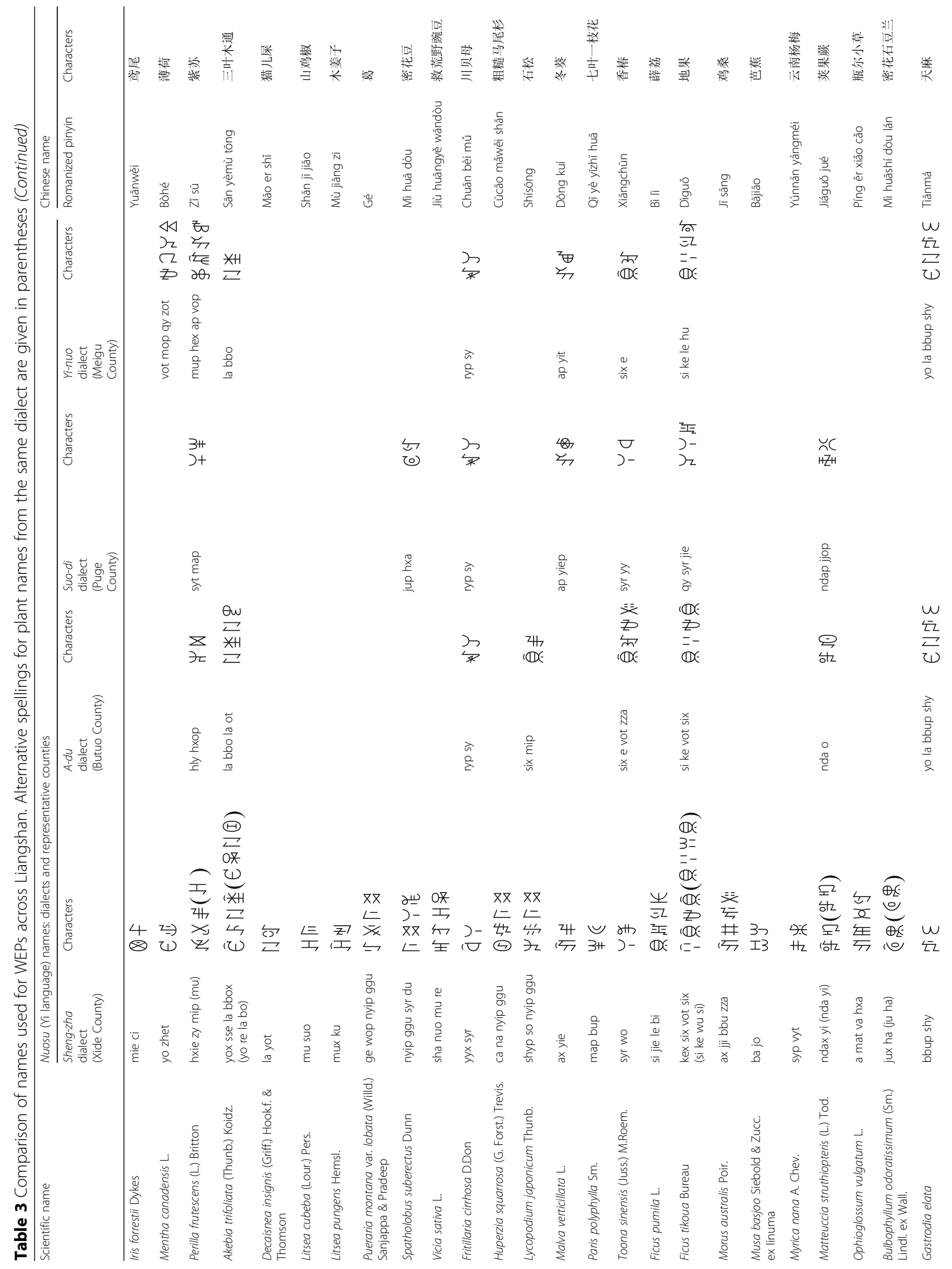




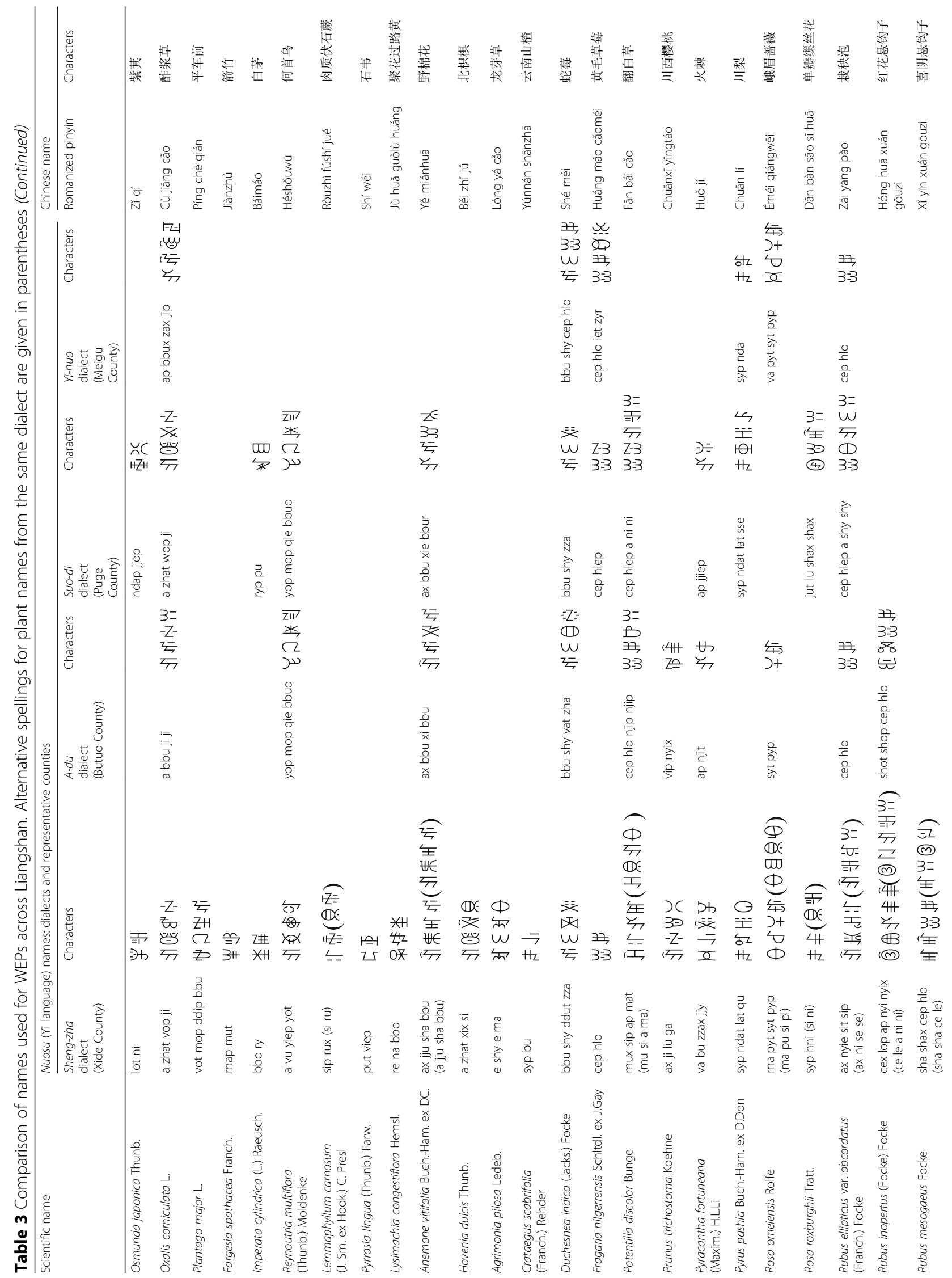




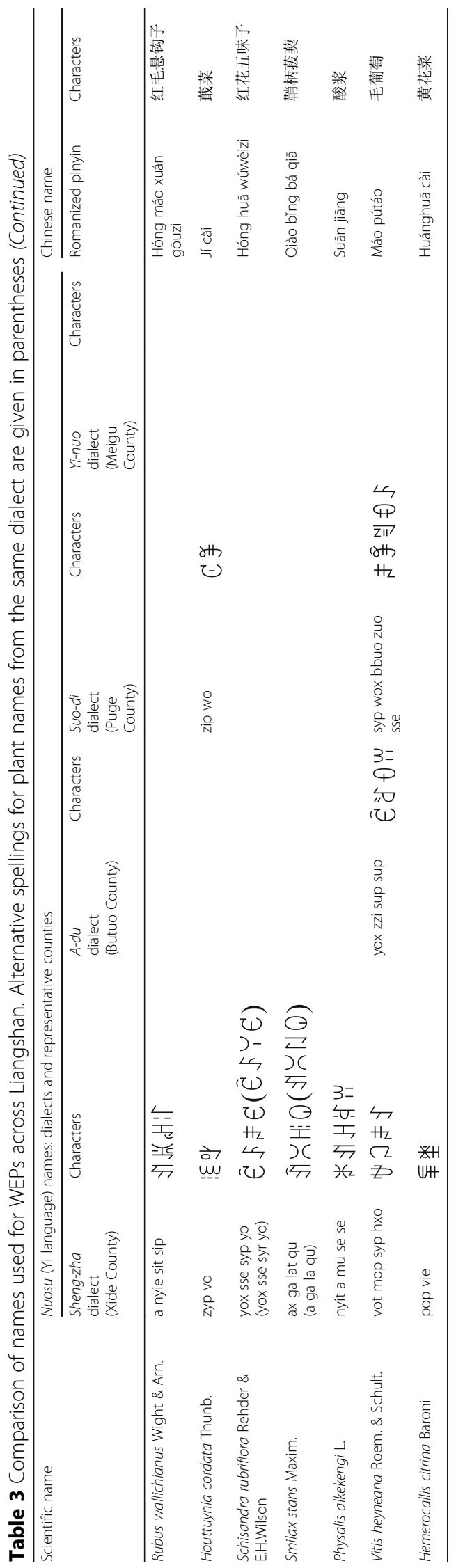


Table 4 The primary consumption patterns of WEPs in Liangshan

\begin{tabular}{|c|c|c|}
\hline $\begin{array}{l}\text { Consumption } \\
\text { pattern }\end{array}$ & $\begin{array}{l}\text { Mode of } \\
\text { consumption }\end{array}$ & Species \\
\hline \multirow[t]{5}{*}{ Primary food } & Boiled in water & $\begin{array}{l}\text { (1) Allium ovalifolium, (2) Amaranthus blitum, (3) Aralia chinensis, (4) Chenopodium hybridum, (5) Fargesia } \\
\text { spathacea, (6) Hemerocallis citrina, (7) Hylocereus undatus, (8) Kalimeris indica, (9) Matteuccia struthiopteris, (10) } \\
\text { Oenanthe javanica, (11) Opuntia ficus-indica, (12) Osmunda japonica, (13) Perilla frutescens, (14) Plantago major, } \\
\text { (15) Pteridium aquilinum, (16) Smilax stans, (17) Sonchus oleraceus, (18) Taraxacum mongolicum, (19) *Toona } \\
\text { sinensis,(20) Toxicodendron vernicifluum, (21) Vicia sativa }\end{array}$ \\
\hline & Made into pickles & $\begin{array}{l}\text { (1) Begonia grandis subsp. sinensis, (2) Nasturtium officinale, (3) Oenanthe javanica, (4) Rorippa dubia, (5) Smilax } \\
\text { stans }\end{array}$ \\
\hline & Made into soup & $\begin{array}{l}\text { (1) Capsella bursa-pastoris, (2) *Cardamine tangutorum, (3) Helwingia japonica, (4) Nasturtium officinale, (5) } \\
\text { Rorippa dubia }\end{array}$ \\
\hline & Raw as salad greens & (1) *Allium macrostemon, (2) *Houttuynia cordata \\
\hline & $\begin{array}{l}\text { Stewed with pork or } \\
\text { chicken }\end{array}$ & $\begin{array}{l}\text { (1) *Angelica sinensis, (2) *Arctium lappa, (3) *Cirsium shansiense, (4) *Codonopsis pilosula subsp. tangshen, (5) } \\
\text { Dioscorea polystachya, (6) *Eucommia ulmoides, (7) *Gastrodia elata, (8) *Imperata cylindrica, (9) *Musa basjoo, } \\
\text { (10) *Ophioglossum vulgatum, (11) *Polygonatum cyrtonema }\end{array}$ \\
\hline \multirow[t]{2}{*}{ Famine food } & Food supplement & $\begin{array}{l}\text { (1) Anemone vitifolia, (2) Artemisia capillaris, (3) Celosia argentea, (4) Pseudognaphalium affine, (5) Pyracantha } \\
\text { fortuneana }\end{array}$ \\
\hline & Starch extraction & (1) Pteridium aquilinum \\
\hline \multirow[t]{2}{*}{ Snack } & Eaten raw & $\begin{array}{l}\text { (1) Actinidia kolomikta, (2) Akebia trifoliata, (3) Berberis jamesiana, (4) Cornus kousa subsp. chinensis, (5) } \\
\text { Crataegus scabrifolia, (6) Decaisnea insignis, (7) Diospyros lotus, (8) Duchesnea indica, (9) Elaeagnus pungens, } \\
\text { (10) Ficus pumila, (11) Ficus tikoua, (12) Fragaria nilgerrensis, (13) Hovenia dulcis, (14) Mahonia bealei, (15) Morus } \\
\text { australis, (16) Metaplexis japonica, (17) Morus austrails, (18) Myrica nana, (19) Musa basjoo, (20) Opuntia ficus- } \\
\text { indica, (21) Oxalis corniculata, (22) Physalis alkekengi, (23) Prunus trichostoma, (24) Pueraria montana var. } \\
\text { lobata, (25) Pyracantha fortuneana, (26) Pyrus pashia, (27) Rosa omeiensis, (28) Rosa roxburghii, (29) Rubus } \\
\text { ellipticus var. obcordatus, (30) Rubus inopertus, (31) Rubus mesogaeus, (32) Rubus wallichianus, (33)Sambucus } \\
\text { adnata, (34) *Schisandra rubriflora, (35) Vaccinium fragile, (34) Viburnum betulifolium, (36) Vitis heyneana }\end{array}$ \\
\hline & Roasted or cooked & (1) Dioscorea polystachya, (2) Quercus schottkyana \\
\hline \multirow[t]{2}{*}{ Spice } & Seasoning & $\begin{array}{l}\text { (1) Allium macrostemon, (2) Houttuynia cordata, (3) Litsea cubeba, (4) Litsea pungens, (5) Mentha canadensis, (6) } \\
\text { Perilla frutescens }\end{array}$ \\
\hline & Sour flavor enhancer & (1) Berberis jamesiana, (2) Viburnum betulifolium, (3) Oxalis corniculata \\
\hline \multirow{2}{*}{$\begin{array}{l}\text { Culinary } \\
\text { coagulant }\end{array}$} & Making cheese & (1) Reynoutria multiflora \\
\hline & Making tofu & (2) Berberis jamesiana \\
\hline \multirow[t]{5}{*}{ Medicine } & Eaten raw & (1) Fritillaria cirrhosa, (2) Gastrodia elata \\
\hline & External use & (1) Artemisia capillaris, (2) Fritillaria cirrhosa, (3) Opuntia ficus-indica, (4) Paris polyphylla \\
\hline & Medicinal soup & (1) Leycesteria formosa, (2) Malva verticillata, (3) Sambucus adnata, (4) Sambucus williamsii \\
\hline & Medicinal tea & $\begin{array}{l}\text { (1) Agrimonia pilosa, (2) Berberis jamesiana, (3) Bulbophyllum odoratissimum, (4) Cynoglossum amabile, (5) } \\
\text { Eclipta prostrata, (6) Huperzia squarrosa, (7) Imperata cylindrica, (8) Incarvillea diffusa, (9) Iris forrestii, (10) } \\
\text { Kalimeris indica, (11) Lemmaphyllum carnosum, (12) Lycopodium japonicum, (13) Lysimachia congestiflora, (14) } \\
\text { Mahonia bealei, (15) Mentha canadensis, (16) Plantago major, (17) Potentilla discolor, (18) Pyrrosia lingua, (19) } \\
\text { Spatholobus suberectus, (20) Taraxacum mongolicum, (21) Trichosanthes kirilowii }\end{array}$ \\
\hline & Tincture & $\begin{array}{l}\text { (1) Araiostegia divaricata var. formosana, (2) Aristolochia versicolor, (3) Crataegus scabrifolia, (4) Eclipta prostrata, } \\
\text { (5) Equisetum giganteum, (6) Gastrodia elata, (7) Hovenia dulcis, (8) Lysimachia congestiflora, (9) Reynoutria } \\
\text { multiflora, (10) Paris polyphylla, (11) Potentilla discolor }\end{array}$ \\
\hline \multirow[t]{5}{*}{ Other use } & Hedge & (1) Pyracantha fortuneana \\
\hline & Honey collection & (1) Pseudognaphalium affine \\
\hline & Kindling & (1) Pseudognaphalium affine \\
\hline & Rituals & (1) Aralia chinensis, (2) Artemisia capillaris, (3) Sambucus adnata, (4) Toxicodendron vernicifluum \\
\hline & Silver jewelry polish & (1) Oxalis corniculata \\
\hline
\end{tabular}

goat's milk. The milk then solidifies, and its smell is effectively masked. It can be eaten directly or made into cheese that can be preserved longer and is considered more delicious than fresh milk. To prepare the cheese, fern leaves are placed on the top and bottom of the milk block, then most of the water is squeezed out with a spoon. Similarly, the Yi people put the juice of Berberis jamesiana into soy milk, and the soy milk solidifies and becomes tofu. 


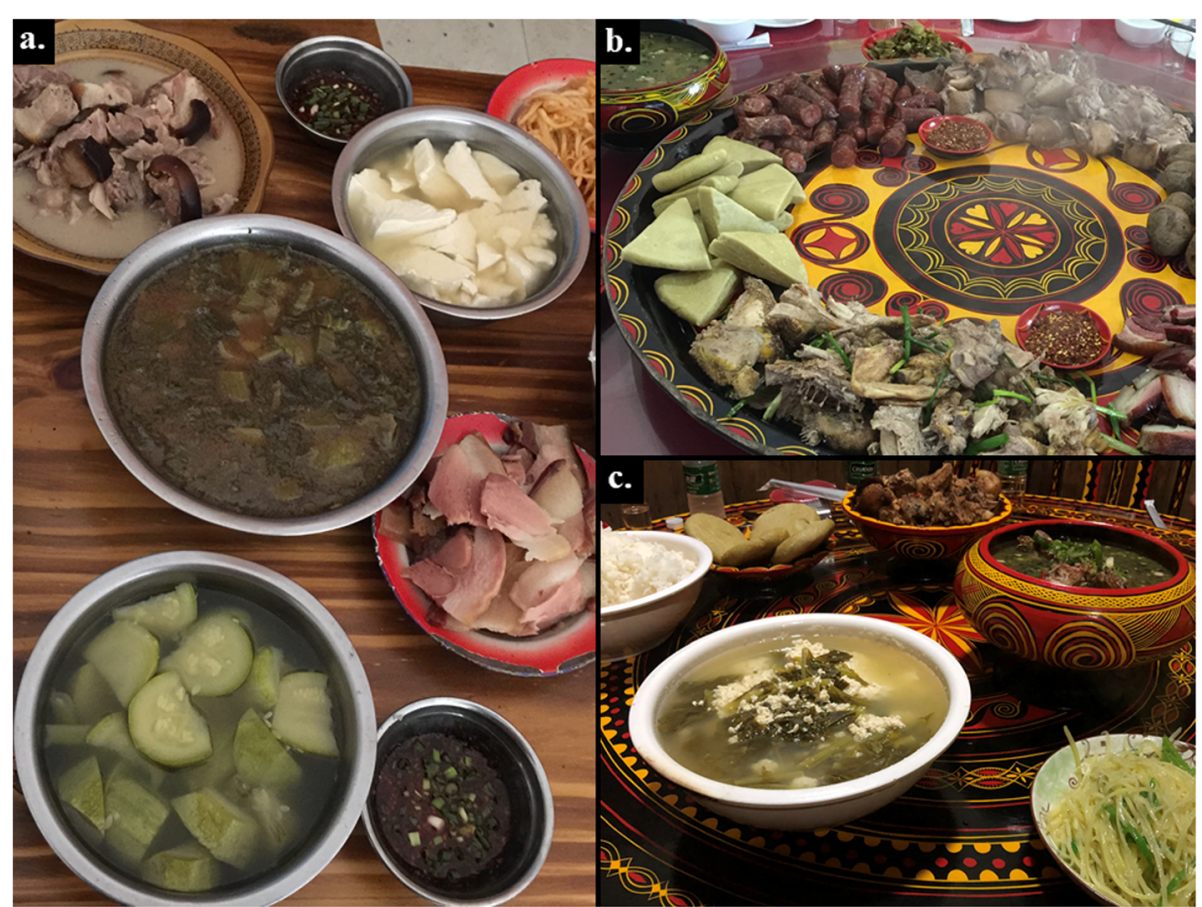

Fig. 2 Typical examples of Liangshan Yi cuisine. a Suäncài tāng or pickle soup (center bowl; Zhaojue County). b From top left corner clockwise: Suäncài tāng (pickle soup), sausage, tuótuó ròu (lump pork), boiled potatoes, ham, tuótuó ji (lump chicken), and bitter buckwheat cakes (Butuo County). c A meal with two types of Suäncài tāng (pickle soup), tuótuó ròu (lump pork), and bitter buckwheat cakes (Zhaojue County). Photo credits: $\mathbf{a}, \mathbf{b} \mathrm{JW} ; \mathbf{c} B C S$

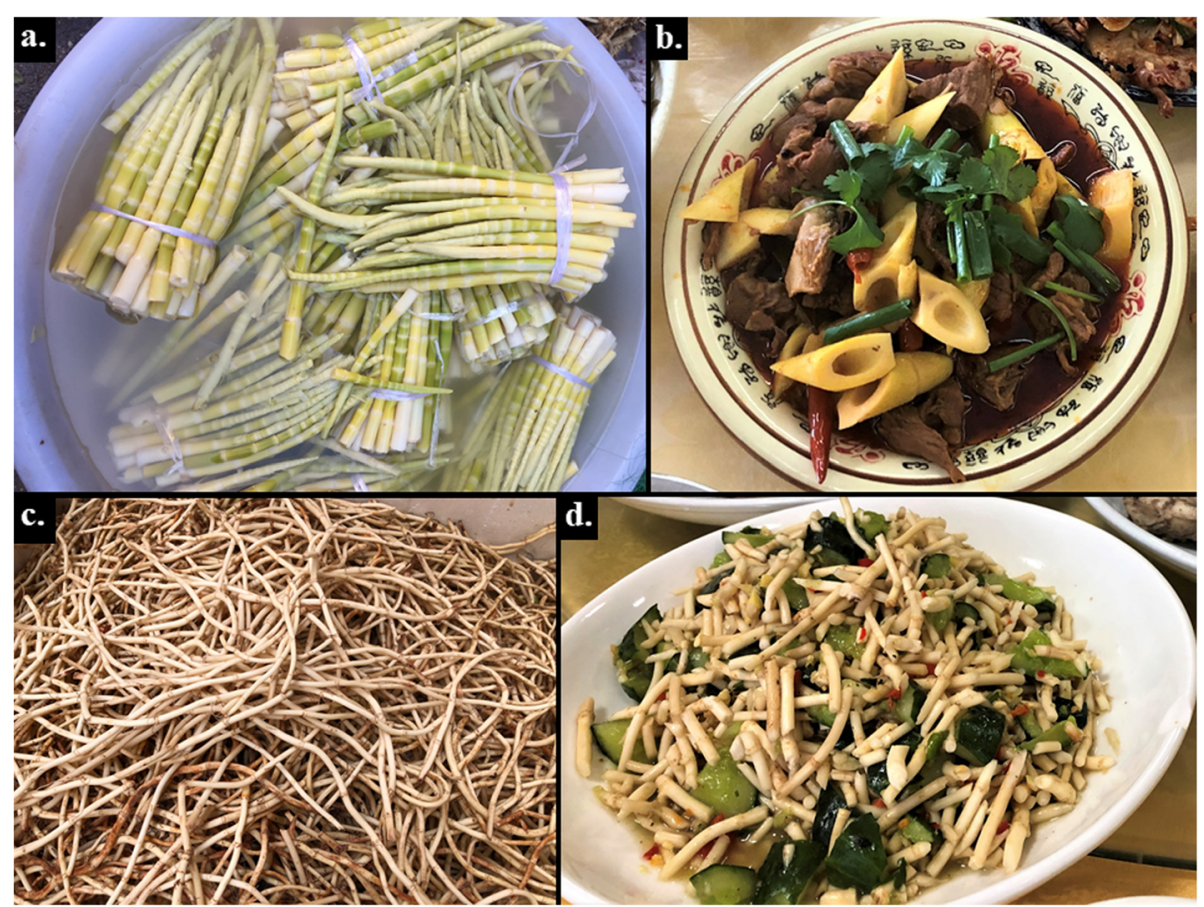

Fig. 3 Before and after. $\mathbf{a}, \mathbf{b}$ The young shoots of bamboo (Fargesia spathacea) are used in many dishes, including bamboo shoots and braised beef (Xichang City). c, d The chopped roots of Houttuynia cordata are used in many Liangshan Yi dishes, including in this cold salad (Puge County). Photo credits: a JW; b-d BCS 


\section{Medicinal edible plants}

According to our survey, the Liangshan Yi use at least 49 species of medicinal edible plants. Of these, several of them have multiple medical applications, with 21 species used to make medicinal tea, 11 species for tinctures, 4 species each for medicinal soups or used externally, and 2 species consumed raw as medicine. Overall, including 13 non-overlapping species from the 15 used as primary foods (the "Primary foods" section) or snacks (the "Snack" section) with secondary medicinal effects, the Liangshan Yi use WEPs to treat 27 ailments (Table 5), including cough, diarrhea, injury, rheumatism, and headaches. There are 10 species used to treat cough, 8 each for treating diarrhea and for tonification, 5 to treat injuries, and 4 each for treating rheumatism and headache.
The WEPs used as medicinal teas are prepared by putting the plant materials into boiling water for about $5-10$ min. For example, the aboveground parts of Agrimonia pilosa, the whole plant of Potentilla discolor, and the roots of Mahonia bealei and Berberis jamesiana are used to cure diarrhea; the roots of Imperata cylindrica are used to stop nosebleeds and suppress coughing; and the flowers of Trichosanthes kirilowii are also used to treat cough. The aboveground portion of Incarvillea diffusa prepared as a tea and mixed with honey and rice wine can treat hepatitis. These teas are usually consumed when someone shows symptoms, drinking approximately $500 \mathrm{ml}$ at a time, 3-5 times a day, until the illness is relieved or cured.

Tinctures are made by putting the plant materials in liquor (ethanol concentration of about 50-65\%) and

Table 5 Symptoms treated by medicinal WEPs among the Liangshan Yi

\begin{tabular}{|c|c|c|c|}
\hline Number & Symptom & Species & Medical plants (no. of informants) \\
\hline 1 & Bone fracture & 2 & Sambucus adnata (142), Sambucus williamsii (207) \\
\hline 2 & Cold & 1 & Equisetum giganteum (56) \\
\hline 3 & Cough & 10 & $\begin{array}{l}\text { Bulbophyllum odoratissimum (100), Crataegus scabrifolia (45), Eclipta prostrata (89), Fritillaria cirrhosa (258), Imperata } \\
\text { cylindrica (112), Iris forrestii (178), Lemmaphyllum carnosum (97), Plantago major (105), Taraxacum mongolicum } \\
\text { (236), Trichosanthes kirilowii (114) }\end{array}$ \\
\hline 4 & Delivery & 1 & Malva verticillata (270) \\
\hline 5 & Detoxification & 1 & Arctium lappa (78) \\
\hline 6 & Diarrhea & 8 & $\begin{array}{l}\text { Agrimonia pilosa (179), Berberis jamesiana (126), Eclipta prostrata (153), Kalimeris indica (34), Mahonia bealei (53), } \\
\text { Plantago major (56), Potentilla discolor (201), Toona sinensis (23) }\end{array}$ \\
\hline 7 & Dyspepsia & 1 & Houttuynia cordata (34) \\
\hline 8 & Enteritis & 1 & Cynoglossum amabile (198) \\
\hline 9 & Gallstone & 1 & Codonopsis pilosula subsp. tangshen (15), Lysimachia congestiflora (220), Pyrrosia lingua (138) \\
\hline 10 & Gastroenteritis & 1 & Aristolochia versicolor (12) \\
\hline 11 & Gastropathy & 3 & Allium macrostemon (27), Huperzia squarrosa (35), Potentilla discolor (137) \\
\hline 12 & Headache & 4 & Aristolochia versicolor (38), Equisetum giganteum (78), Gastrodia elata (134), Reynoutria multiflora (159) \\
\hline 13 & Heart disease & 2 & Musa basjoo (109), Spatholobus suberectus (89) \\
\hline 14 & Hemorrhoid & 1 & Cynoglossum amabile (107) \\
\hline 15 & Hepatitis & 1 & Incarvillea diffusa (214) \\
\hline 16 & Hypertension & 2 & Araiostegia divaricata var. formosana (67), Cardamine tangutorum (56) \\
\hline 17 & Hyperthermia & 1 & Mentha canadensis (43) \\
\hline 18 & Injury & 5 & $\begin{array}{l}\text { Aristolochia versicolor (52), Artemisia capillaris (156), Fritillaria cirrhosa (189), Lemmaphyllum carnosum (34), Paris } \\
\text { polyphylla (253) }\end{array}$ \\
\hline 19 & Measles & 1 & Leycesteria formosa (132) \\
\hline 20 & Muscle pain & 1 & Paris polyphylla (121) \\
\hline 21 & Nephropathy & 2 & Eucommia ulmoides (89), Cirsium shansiense (62) \\
\hline 22 & Nosebleed & 1 & Imperata cylindrica (45) \\
\hline 23 & Pneumonia & 1 & Eclipta prostrata (54) \\
\hline 24 & Rheumatism & 4 & Allium macrostemon (27), Huperzia squarrosa (78), Lycopodium japonicum (95), Sambucus adnata (53) \\
\hline 25 & Stomachache & 1 & Equisetum giganteum (74) \\
\hline 26 & Tonification & 8 & $\begin{array}{l}\text { Angelica sinensis (135), Arctium lappa (93), Cirsium shansiense (126), Codonopsis pilosula subsp. tangshen (56), } \\
\text { Hovenia dulcis (75), Ophioglossum vulgatum (108), Polygonatum cyrtonema (167), Schisandra rubriflora (75) }\end{array}$ \\
\hline 27 & Tonsillitis & 1 & Opuntia ficus-indica (21) \\
\hline
\end{tabular}


waiting at least half a month before drinking. For treatment, about $50-100 \mathrm{ml}$ is consumed two to three times a day until symptoms subside. For example, Paris polyphylla tinctures are used to cure injuries (e.g., bruises caused by a fall or sprains), Potentilla discolor tinctures are used to treat diarrhea and gastropathy, Gastrodia elata tinctures are used to relieve headaches, and Lysimachia congestiflora tinctures are used to treat gallstones.

To make medicinal soups, the plant materials are cooked for a while in a fried egg soup, then the patient consumes the soup. For example, the egg soup of Malva verticillata can be used to aid childbirth, the egg soups of Sambucus adnata and S. williamsii can help heal bone fractures, and the egg soup of Leycesteria formosa can cure measles.

Of the two species of WEPs eaten raw as medicine, Fritillaria cirrhosa bulbs are collected, sun dried, and stored. The bulbs are then crushed into a powder and ingested orally to treat cough. The powder of dried Gastrodia elata rhizomes can be eaten directly to relieve headache.

For the plants used externally, the powder of Fritillaria cirrhosa and the ground pulp of Paris polyphylla or Artemisia capillaris can be applied directly to trauma wounds to quickly stop the bleeding and help wounds heal more rapidly. Some Yi cut the prickly pear cactus's epidermis (Opuntia ficus-indica) and put it on the cheek to treat tonsillitis. It is, however, important to note there are many other medicinal plants used externally by the Liangshan Yi that are not recorded here because they are not WEPs.

\section{Other uses}

We found that seven species of WEPs also had other noneating uses. For example, four species are used as ritual plants. Artemisia capillaris is used in cleansing rituals. Heated stones are placed in a container with $A$. capillaris and water, causing a white steam to arise filled with the plant's aromatic oil. The person or thing passing through the steam is considered ceremonially clean. The branches of Aralia chinensis are used in the ritual of installing ancestral spirits (i.e., assisting the souls of deceased relatives to reach the spiritual realm). The branches of both Toxicodendron vernicifluum and Sambucus adnata are used in exorcism rituals.

Liangshan Yi collect and dry Pseudognaphalium affine as a type of kindling. Some elder Yi often carry steel, flint, and kindling in their pouches. When they want to smoke, they strike the steel against the flint to generate a spark, setting the prepared kindling on fire to ignite the pipe. Secondly, when Yi hunters find a wild beehive, in order to collect honey, the dried vegetation of $P$. affine is ignited under the hive, and the resulting smoke dispels the bees and/or stuns them, so that the hunter can easily obtain the honey. Yi women use Oxalis corniculata to scrub, de-tarnish, and polish silver jewelry (earrings, rings, bracelets, etc.), which are important items in Yi traditional attire (Figs. 4 and 5). Pyracantha fortuneana is a very common hedge plant and is planted around the yard perimeters in many Yi villages.

\section{Months of collection}

The Liangshan Yi mainly collect WEPs from March to October (Fig. 6). The greatest number of species can be collected in August (95 species), followed by May (91), September and October (90 each), July (87), April (86), June (85), and March (78). Due to inclement weather and frost, the number of WEPs collected during late autumn and winter is much fewer. For instance, only 34 species are collected in November, 29 species in December, 24 species in February, and only 23 species in January.

Most of the WEPs used for food are collected from March to June, with the total number gradually declining through October, with only four species collected yearround. Wild fruits consumed as snacks as well as spices are most often collected from July to October. Medicinal plants are mainly collected from March to October. For culinary coagulants, the leaves of Reynoutria multiflora

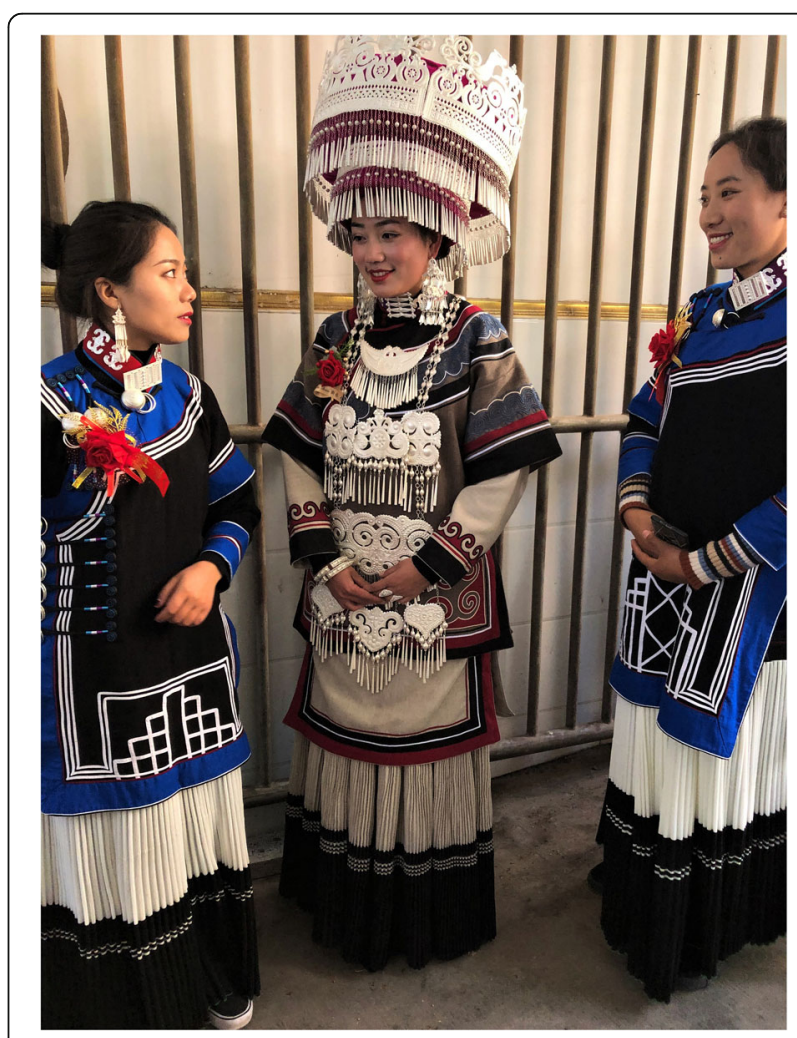

Fig. 4 A young bride on her wedding day (Puge County). The bride (center) and bridesmaids are donning traditional silver Yi jewelry and other handicrafts; aboveground portions of Oxalis corniculata are traditionally used to de-tarnish and polish the silver attire. Fern fiddleheads are depicted on both the bride's silver filigree and embroidered dress (e.g., sleeves and waist). Photo credit: BCS 


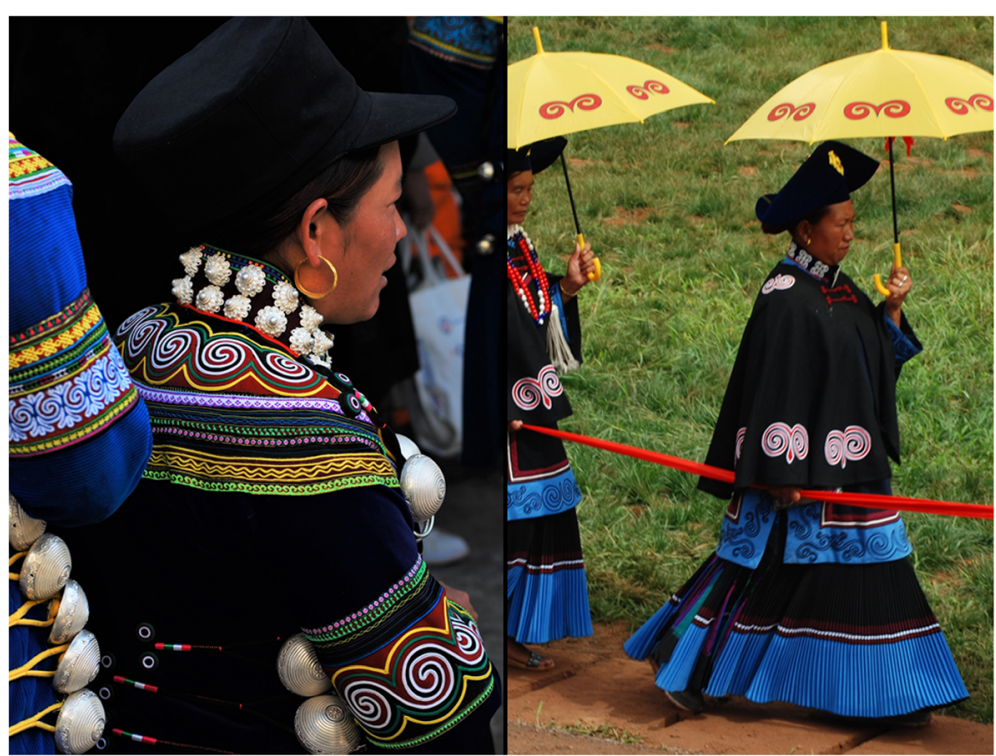

Fig. 5 Fern fiddleheads, representing fertility and abundance, are frequently depicted on Liangshan Yi women's traditional attire. Puge County (left) and Xide County (right). Photo credits: BCS

are collected all year-round, but the fruits of Berberis jamesiana are only collected when mature (September to December). All seven WEPs in the "other uses" category are collected year-round.

\section{Commercial valuation}

Many Liangshan Yi collect certain species of WEPs to sell at the market to augment their household incomes. We found 35 species of WEPs sold in the markets we surveyed, with 8 species sold as food, 18 species as medicinal herbs, 6 species as wild fruits (including the roots of Pueraria montana var. lobata eaten as a snack), and 3 species as spices (Table 2).

The prices of medicinal WEPs ( $¥ 40-2000 \mathrm{RMB} / \mathrm{kg}$; note: $\$ 1 \mathrm{USD}=¥ 6.8 \mathrm{RMB}$ ) were much higher than the price of foods and fruits ( $¥ 5-20 \mathrm{RMB} / \mathrm{kg}$; Table 2). Fritillaria cirrhosa, Paris polyphylla, Gastrodia elata, Reynoutria multiflora, Angelica sinensis, Spatholobus suberectus, Huperzia squarrosa, and Ophioglossum vulgatum were targets of commercial acquisition, so their prices were particularly high. For example, dry Fritillaria cirrhosa sold for $¥ 2000 \mathrm{RMB} / \mathrm{kg}$ and Paris polyphylla sold for $¥ 600 \mathrm{RMB} / \mathrm{kg}$. Fresh Gastrodia

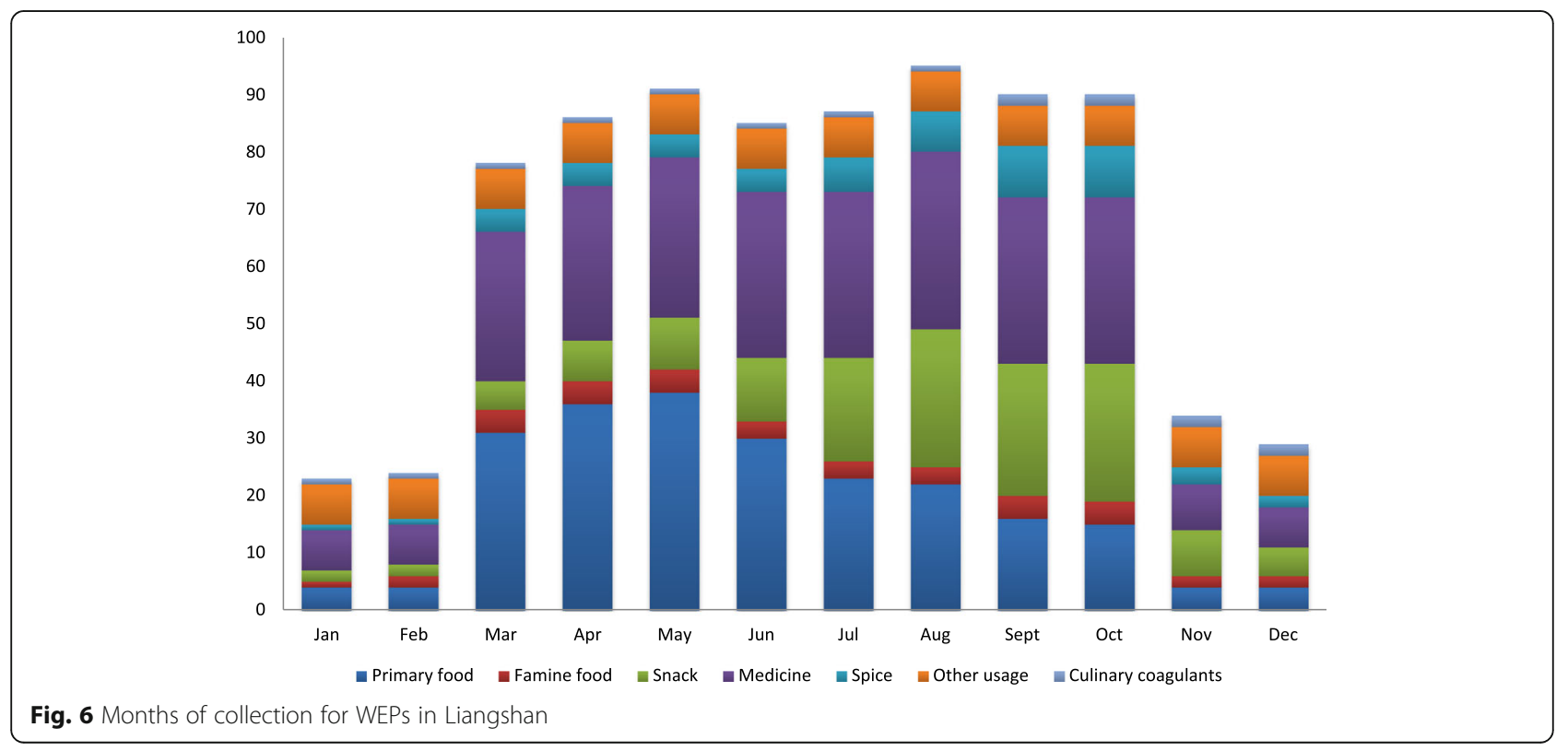


elata sold for approximately $¥ 100 \mathrm{RMB} / \mathrm{kg}$, while the dried form sold for $¥ 500 \mathrm{RMB} / \mathrm{kg}$. In contrast, because of their ample supply and wide distribution, the price of Taraxacum mongolicum and Plantago major was much lower (¥10 $\mathrm{RMB} / \mathrm{kg})$.

Fritillaria cirrhosa and Paris polyphylla are important raw materials for many traditional Chinese medicine (TCM) preparations. F. cirrhosa is a key herb used to make a popular cough syrup called Chuān bèi pípá gāo (川贝枇杷膏), which is used by the Chinese diaspora worldwide, including in East Asia, Europe, and North America. F. cirrhosa is also one of the most important ingredients in a couple famous Chinese proprietary medicines, including Yúnnán Báiyào (云南白药), used for treating injuries and stopping bleeding, and Gōng Xuè Ning (宫血宁), used for curing excessive menstruation. It is well-documented that $P$. polyphylla has near-miraculous medicinal properties to cure wounds. External application of the powdered roots can rapidly stop bleeding, reduce inflammation, and even treat venomous snake bites [53-56].

Since the 1980s, many pharmaceutical companies began establishing local branches in Liangshan to acquire Paris polyphylla. Consequently, interviewees reported that the wild resources of this plant have been so greatly deplenished that it is now very difficult to collect them for local medical needs. Therefore, many locals in Liangshan have begun purchasing wild seedlings of $P$. polyphylla to plant in their courtyards for convenient access when someone is wounded (Fig. 7). They will also sell them after they have grown to maturity. During one of our field surveys in Liangshan (Meigu County, April 2017), we met a local team whose sole aim was to collect wild P. polyphylla seedlings. They said that about 2000 individuals could be collected each day, and each plant could be sold to villagers for about $¥ 0.2$ RMB.

\section{Use value}

The five species with the highest use values (UVs) were Berberis jamesiana (1.92), Pyracantha fortuneana (1.87), Artemisia capillaris (1.44), Pteridium aquilinum (1.35), and Houttuynia cordata (1.26). The species with the lowest UVs were Lycopodium japonicum (0.24), Spatholobus suberectus (0.22), Huperzia squarrosa (0.20), Araiostegia divaricata var. formosana (0.17), and Aristolochia versicolor (0.13) (Tables 2 and 6).

\section{Discussion}

\section{Yi Culture of WEPs in Liangshan}

Although the Liangshan Yi people have their own traditional written language, historically, it was not widely learned by the general public. Instead, it was primarily used only by their traditional ritual specialists, the bimox. With the broader Yi population being largely illiterate throughout much of Liangshan's history, their cultural knowledge and traditional customs were instead transmitted through oral communication

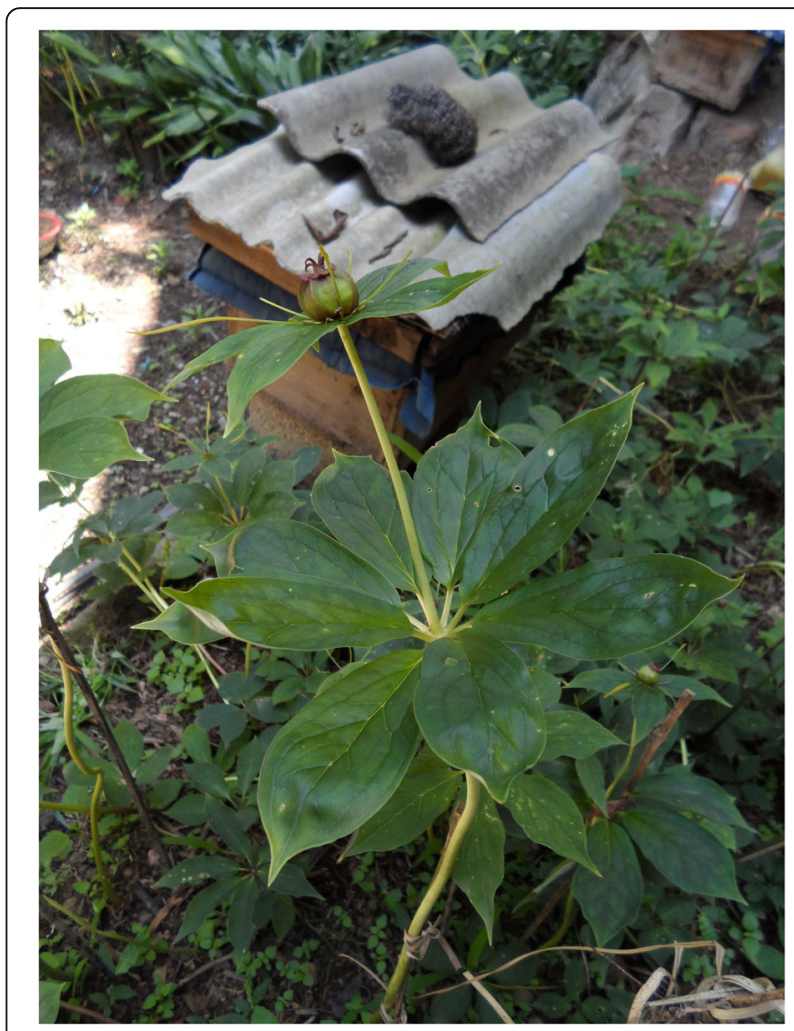

Fig. 7 Young plants of Paris polyphylla are grown in the courtyards of homes throughout Liangshan, here in Puge County. Photo credit: BCS

techniques [57], including knowledge of WEPs. One way this information was organized for easy memory and transmission was through traditional Yi cultural sayings. These sayings often included information about the WEPs' flavors, proper collection times, and medical uses. For example, one traditional saying about the good flavor of soup made from Cardamine tangutorum says "I should share the vegetable of Cardamine tangutorum with my mom but not the soup"

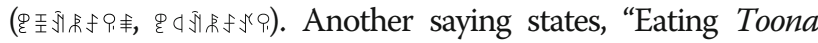
sinensis prevents diarrhea if collected before the cuckoo

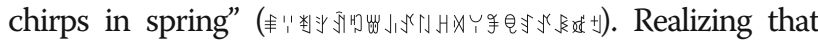
Houttuynia cordata has the effect of promoting rapid digestion, a traditional Yi saying warns not to eat it during times of famine. It states, "a satiated people will be hungry soon after eating Houttuynia cordata, but hungry people never try it because they will starve" (

\section{Climate, lacto-fermentation, and collection season}

In contrast to the Han people, who primarily settled in river valleys, most Yi people live in mountainous areas in Liangshan, with limited arable land, colder weather, and longer winters, so this has greatly influenced their cultural traditions, preferred WEPs, and associated knowledge. Since there is a shorter time period to cultivate crops and vegetables, to ensure year-round food 
Table 6 WEPs with the highest and lowest use values (UVS)

\begin{tabular}{|c|c|c|c|c|c|c|c|c|}
\hline & Family & Scientific name & $\begin{array}{l}\text { Usage } 1 \\
\text { (frequency) }\end{array}$ & $\begin{array}{l}\text { Usage } 2 \\
\text { (frequency) }\end{array}$ & $\begin{array}{l}\text { Usage } 3 \\
\text { (frequency) }\end{array}$ & $\begin{array}{l}\text { Usage } 4 \\
\text { (frequency) }\end{array}$ & $\Sigma U s$ & UVs \\
\hline \multirow[t]{10}{*}{$\begin{array}{l}\text { Species with the } \\
\text { highest UVs }\end{array}$} & Berberidaceae & $\begin{array}{l}\text { Berberis jamesiana Forrest \& } \\
\text { W.W.Sm. }\end{array}$ & Snack (314) & Spice (209) & $\begin{array}{l}\text { Medicine } \\
(126)\end{array}$ & $\begin{array}{l}\text { Culinary } \\
\text { coagulant (112) }\end{array}$ & 761 & 1.92 \\
\hline & Rosaceae & $\begin{array}{l}\text { Pyracantha fortuneana (Maxim.) } \\
\text { H.L.Li }\end{array}$ & Snack (379) & $\begin{array}{l}\text { Other use } \\
\text { (196) }\end{array}$ & $\begin{array}{l}\text { Famine food } \\
\text { (166) }\end{array}$ & & 741 & 1.87 \\
\hline & Compositae & Artemisia capillaris Thunb. & $\begin{array}{l}\text { Other use } \\
\text { (346) }\end{array}$ & $\begin{array}{l}\text { Medicine } \\
\text { (156) }\end{array}$ & $\begin{array}{l}\text { Famine food } \\
\text { (67) }\end{array}$ & & 569 & 1.44 \\
\hline & Dennstaedtiaceae & Pteridium aquilinum (L.) Kuhn & $\begin{array}{l}\text { Primary food } \\
\text { (379) }\end{array}$ & $\begin{array}{l}\text { Famine food } \\
\text { (157) }\end{array}$ & & & 536 & 1.35 \\
\hline & Saururaceae & Houttuynia cordata Thunb. & $\begin{array}{l}\text { Primary food } \\
\text { (357) }\end{array}$ & Spice (109) & $\begin{array}{l}\text { Medicine } \\
\text { (34) }\end{array}$ & & 500 & 1.26 \\
\hline & Oxalidaceae & Oxalis corniculata L. & Snack (250) & Spice (198) & $\begin{array}{l}\text { Other use } \\
\text { (13) }\end{array}$ & & 461 & 1.16 \\
\hline & Compositae & Cirsium shansiense Petr. & $\begin{array}{l}\text { Primary food } \\
\text { (326) }\end{array}$ & $\begin{array}{l}\text { Medicine } \\
(126)\end{array}$ & & & 452 & 1.14 \\
\hline & Lamiaceae & Perilla frutescens (L.) Britton & Spice (289) & $\begin{array}{l}\text { Primary food } \\
\text { (149) }\end{array}$ & & & 438 & 1.11 \\
\hline & Orchidaceae & Gastrodia elata Blume & $\begin{array}{l}\text { Medicine } \\
(296)\end{array}$ & $\begin{array}{l}\text { Primary food } \\
\text { (134) }\end{array}$ & & & 430 & 1.09 \\
\hline & Adoxaceae & Sambucus adnata Wall. ex DC. & Snack (235) & $\begin{array}{l}\text { Medicine } \\
(142)\end{array}$ & $\begin{array}{l}\text { Other use } \\
(45)\end{array}$ & & 422 & 1.07 \\
\hline \multirow[t]{10}{*}{$\begin{array}{l}\text { Species with the } \\
\text { lowest UVs }\end{array}$} & Helwingiaceae & Helwingia japonica (Thunb.) F.Dietr. & $\begin{array}{l}\text { Primary food } \\
\text { (105) }\end{array}$ & & & & 105 & 0.27 \\
\hline & Equisetaceae & Equisetum giganteum L. & $\begin{array}{l}\text { Medicine } \\
(102)\end{array}$ & & & & 102 & 0.26 \\
\hline & Orchidaceae & $\begin{array}{l}\text { Bulbophyllum odoratissimum (Sm.) } \\
\text { Lindl. ex Wall. }\end{array}$ & $\begin{array}{l}\text { Medicine } \\
(100)\end{array}$ & & & & 100 & 0.25 \\
\hline & Amaranthaceae & Celosia argentea L. & $\begin{array}{l}\text { Famine Food } \\
\text { (99) }\end{array}$ & & & & 99 & 0.25 \\
\hline & Cactaceae & $\begin{array}{l}\text { Hylocereus undatus (Haw.) Britton } \\
\text { \& Rose }\end{array}$ & $\begin{array}{l}\text { Primary food } \\
\text { (98) }\end{array}$ & & & & 98 & 0.25 \\
\hline & Lycopodiaceae & Lycopodium japonicum Thunb. & $\begin{array}{l}\text { Medicine } \\
\text { (95) }\end{array}$ & & & & 95 & 0.24 \\
\hline & Leguminosae & Spatholobus suberectus Dunn & $\begin{array}{l}\text { Medicine } \\
\text { (89) }\end{array}$ & & & & 89 & 0.22 \\
\hline & Lycopodiaceae & $\begin{array}{l}\text { Huperzia squarrosa (G. Forst.) } \\
\text { Trevis. }\end{array}$ & $\begin{array}{l}\text { Medicine } \\
(78)\end{array}$ & & & & 78 & 0.20 \\
\hline & Davalliaceae & $\begin{array}{l}\text { Araiostegia divaricata var. } \\
\text { formosana (Hayata) M. Kato }\end{array}$ & $\begin{array}{l}\text { Medicine } \\
(67)\end{array}$ & & & & 67 & 0.17 \\
\hline & Aristolochiaceae & Aristolochia versicolor S.M.Hwang & $\begin{array}{l}\text { Medicine } \\
(52)\end{array}$ & & & & 52 & 0.13 \\
\hline
\end{tabular}

supplies, the Liangshan Yi preserve vegetables through lacto-fermentation techniques by making pickles.

Lacto-fermentation is a food preservation technique shared by many other people groups around the world $[58,59]$, as well as elsewhere in China, such as Tibetans in Gansu Province [23], who live in northern latitude and high altitude areas with long winters. The Liangshan Yi believe these pickles are not only appetizing but aid their digestion, which is a well-documented benefit of eating lacto-fermented foods as probiotics [58, 59].
These cultural beliefs are also similar to those of the people living in the eastern part of Gilan Province (North Iran), who use pickled sour orange fruits to fortify their stomachs [60]. The overall nutritive value of lacto-fermented foods and drinks is also recognized by diverse people groups across Eastern Europe, Turkey, and the Caucasus [58].

In addition, Liangshan $\mathrm{Yi}$ cuisine is heavily soupbased, because the staple food is dense, highcarbohydrate buckwheat cakes, which require soup to 
help swallow (Fig. 2). Consequently, a traditional Yi proverb states, "Soup can nourish people for seven days,

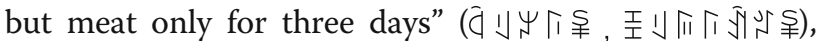
indicating their preference for soup [61]. Nevertheless, the pickle soup, a quintessential component of the Liangshan Yi people's traditional diet finds a cultural parallel with the Eastern European (e.g., Poland, Lithuania, and Belarus) tradition of preparing sour pickle soups from the lacto-fermented shoots and leaves of hogweed (Heracleum sphondylium) [58].

We found that the collection dates for most WEPs are concentrated between March and October. The collection of tender shoots, leaves, and aboveground plant parts for food most often occur between March and June, but, as we predicted, the collection of wild edible fruits (food, snack, and spice categories) tend to be collected later in the growing season (summer and autumn). Due to the seasonal ontogeny in high-elevation Liangshan, these collection seasons are similar to those recorded elsewhere in Northern Hemisphere temperate [22] and high-elevation subtropical regions [4, 5]. However, the collection of famine foods, culinary coagulants, and the most valuable medicinal plants, driven more by the interests of necessity, weather, and economics, were less closely related to the growth and reproduction characteristics of the WEPs. Similarly, the collection of all seven "other use" WEPs (including all four ritual plants) is not affected by season, because the parts collected are mostly branches.

\section{WEP diversity, use values, and cultural significance}

In keeping with our expectations, the families Rosaceae (14 species) and Compositae (8 species) had the highest representation of species in this study, but Lamiaceae was only represented by 2 species (Table 2). The greater number of collected WEPs from these families is similar to the patterns documented elsewhere [5, 22, 23, 62], but unlike those studies, other common families (e.g., Asparagaceae, Caprifoliaceae, Liliaceae, and Polygonaceae) were each represented by a single species in our study. This suggests that the relative abundance of a family within a particular area is not reason enough for its species to be collected for food or medicine. In this sense, although we did not specifically test the theory of non-random plant selection [17, 26], our data lends further support for this widely tested theory. For example, if the selection/collection of WEPs were truly random or "placebo" (rather than based on many years of experience and local knowledge of the flora), every common/ abundant family would be expected to have greater representation among the utilized WEPs.

We might also expect that the common families would have more species with greater UVs, but, instead, we found that species from 9 different families had the top 10 highest UVs (Table 6). Although all 3 families (Compositae, Lamiaceae, and Rosaceae) had species within the top 10 highest UVs, only Compositae had more than 1 (\#3 and \#6). However, this may be due to the particularly high species diversity in Liangshan, in that despite stiff competition, these species have proven over time to be the most versatile [26, 63]. Nevertheless, when looking at plant UVs, the relative cultural importance of the collective taxa from each family is better appreciated. For example, of the top $20 \mathrm{UVs}$ (Table 2), the proportion of taxa from the 3 families increases: Compositae (3/8 taxa; 37.5\%), Lamiaceae $(2 / 2$ taxa; $100 \%)$, and Rosaceae $(6 / 14 ; 42.9 \%)$, and of the top 30 UVs: Compositae (4/8 taxa; 50.0\%), Lamiaceae (2/2 taxa; $100 \%)$, and Rosaceae $(9 / 14 ; 64.3 \%)$. So, according to the UVs, a majority of the collected WEPs from all 3 families are among the most culturally important species, and only 2 species (both Compositae) fall within the bottom 30 UVs (\#79 and \#86; Table 2).

As Gaoue et al. [26] point out, plant use values are essential measures of plant versatility. Thus, not only are the high-UV WEPs widely distributed with large populations throughout Liangshan, the Yi people have found these particular species to be very useful in their daily lives. For example, many Yi people since childhood have consumed the fruit of Berberis jamesiana (highest UV) and Pyracantha fortuneana (UV \#2) raw as snacks between meals while collecting firewood or herding. Most adults also know that the juice of $B$. jamesiana can be used as a culinary coagulant to make tofu or as a flavor enhancer (spice) to increase the sourness of pickle soups. Similarly, the dense growth and thorns of $P$. fortuneana, beautiful white flowers, edible red fruit, and long fruiting period make it commonly planted as a hedge.

Nevertheless, historical events and outside influences may have amplified the relative importance of some of these plants. For example, in the 1950s, the National Pharmaceutical Company began commercial acquisition in Liangshan of the root bark of Berberis jamesiana and related species (Berberidaceae) to extract and refine the bioactive compound (berberine), which is medically valuable for treating diarrhea and other ailments. The large volume of acquisitions of $B$. jamesiana root bark for extraction of berberine deeply affected the perception (including the local name) of this plant by many Liangshan Yi [28]. This may contribute to why it has the highest UV in our survey. Similarly, Pyracantha fortuneana is an important famine food remembered by elderly Yi people who had experienced severe famine years. The fruit was ground into pulp and mixed into Tartary buckwheat (Fagopyrum tataricum) flour or cornmeal to make cakes, augmenting the nutrition of the flours and increasing their volumes. 
Our results also demonstrate large differences between the economic/commercial valuation and cultural evaluation (UVs) for particular species (Tables 2 and 6). For example, although we found that medicinal WEPs sell at the market for much higher prices than those used for other purposes, of the three most economically valuable medicinal WEPs, only the use value of Gastrodia elata ( $¥ 100 \mathrm{RMB} / \mathrm{kg}$ wet; $¥ 500 \mathrm{RMB} / \mathrm{kg}$ dry) is in the top ten (UV \#9). In contrast, Fritillaria cirrhosa (¥2000 RMB/ $\mathrm{kg}$ ) and Paris polyphylla (¥600 RMB/kg) were more expensive, but they had much lower cultural significance overall (UV \#44 and \#46, respectively). However, both of these species are only used for medicine, while Gastrodia elata is also an important food plant widely sold in the markets. Incidentally, because of children's good eyesight and flexible bodies, they more easily fit in and around bushes. Thus, during the flowering period of this orchid (when it briefly appears above ground), many Yi children are encouraged to participate in collection activities, so children, in particular, tend to be very knowledgeable about this species.

This demonstrates the need for longitudinal studies to measure plant use values within the same cultural context over time (across differing demographic variables) in order to more robustly test ethnobotanical theories. Cultures are dynamic and resilient, constantly adapting to changing conditions, including the introduction of new species and decline of formerly common species [26]. Unlike the cultural keystone species theory, which is hard to quantify beyond cultural perceptions of foundationally important species [26, 64, 65], plant use values do not seek to measure absolute importance of particular species within a culture, but instead measure their relative cultural importance at a given moment (or "snapshot") in time $[26,66]$. Thus, the rank order of UVs should be interpreted with this in mind.

For example, we found that the tender shoots of Pteridium aquilinum (UV \#4) are widely eaten by the Liangshan Yi as vegetables, but its roots are also used as a famine food. This species is representative of an important cultural reverence more broadly applied by the Yi people to multiple species of ferns, including Matteuccia struthiopteris (UV \#24). For thousands of years, ferns like these have been an important source of regular nourishment, medicine, and famine food for the Liangshan Yi. The ancient Yi scriptures Zuò zhāi xiàn yào gōng shēng jīng (作斋献药供牲经) describe the fern fiddleheads as representative of abundant and prosperous descendants. Therefore, fiddleheads are common decorative motifs on the Liangshan Yi's clothing, textiles, and other material culture items (Figs. 4 and 5), which highlight their cultural veneration and gratitude to ferns for providing food in times of famine. At the same time, these fern motifs are expressions of hope that their children will also flourish like the ferns [67].

\section{Edible medicinal plants and conservation}

As we hypothesized, many of the WEPs primarily consumed by the Liangshan $\mathrm{Yi}$ as food also have medicinal effects. Some of these are intentionally ingested for their healthful effects as part of an overall "healthy diet." For example, the rhizomes of Gastrodia elata (UV \#9), whole plant of Houttuynia cordata (UV \#5), and roots of Cirsium shansiense (UV \#7) are eaten as vegetables, understanding their medicinal effects. This further supports the argument put forward by various authors that there is no clear distinction between the concepts of food and medicine in many cultures $[16,17,19]$. This is also similar to the documented use of medicinal edible plants (e.g., ginger, buckwheat, and bitter melon) as dietary staples among the Yi people of Guizhou Province, particularly in their ancient medical text $Q \grave{\imath}$ gŭ shŭ (启谷署) [68]. In addition, the Yi that live in Xishuangbanna (Lancang River Basin), in southern Yunnan Province, also have the custom of using medicinal edible plants to strengthen their physical health and prevent disease. They collect many medicinal WEPs for meals and stew them with pork every year during the Dragon Boat Festival [69], which is a holiday adopted from the Han Chinese. Similarly, the Yi, Lahu, and Han people in the Simao area of Yunnan Province, as well as the Zhuang people of Guangxi, have related traditions of eating meals of medicinal roots during the Dragon Boat Festival [70].

Nevertheless, our data on medicinal WEPs also reveal some of the most common diseases and general health concerns that afflict the Liangshan $\mathrm{Yi}$ communities (Table 5). In total, taxa from 37 out of the 62 families of WEPs in our study were used for medicine, and, as documented elsewhere $[9,16,71]$, for a given species, the medicinal plant parts often differed from those collected for food or other uses (Table 2). For example, Artemisia capillaris (UV \#3) is an important ritual plant, with its aboveground parts used in almost all cleansing rituals as well as for medicine, but only the tender shoots are used as a famine food. Similarly, the fruit of Sambucus adnata (UV \#10) is used as a snack, but the aboveground portions have medicinal and ritual uses.

We also documented an interesting preparation method utilized by the Liangshan Yi for four medicinal WEPs, in which plant parts are prepared in fried egg soups for ingestion by the patient. This preparation method is intentional and only used for certain plant parts from particular species to treat known ailments. This appears to be similar to the preparation methods of certain medicinal plants ingested by the Yi people of Chuxiong Prefecture in Yunnan Province [72], but more research is required to understand the significance of the egg preparation technique on the bioactivity of these plant compounds.

Seven out of the ten WEPs with the lowest UVs (Tables 2 and 6) are medicinal plants (with high medicinal value 
but less cultural significance overall), and none of them is WEPs traditionally used by the Liangshan Yi, except for Equisetum giganteum and Bulbophyllum odoratissimum. Due to improvements in the transportation and communications infrastructure in the Liangshan region in recent years, the Liangshan Yi now interact with other cultures more frequently and widely than ever before. Consequently, some Yi people have learned about these medicinally valuable species through interacting and trading herbs with the Han Chinese [28].

In recent years, however, the Liangshan Yi have struggled to find certain species of medicinal WEPs as their great economic value has led to commercial exploitation and overharvest, leading to an overall sharp decline in wild populations. For example, commercial acquisition of Paris polyphylla across Sichuan was about $300 \mathrm{t}$ in the 1990s, but as the slow-growing wild populations diminished, the commercial collection declined to less than $100 \mathrm{t}$ by 2010 [73]. The overall quality of the collected WEPs has also reportedly declined. Therefore, the Liangshan Yi people started collecting wild seedlings of certain valuable WEPs to plant in their courtyards. As we found, many Liangshan Yi either began collecting seedlings of Paris polyphylla from the wild or purchasing seedlings from collecting teams in order to plant in their courtyards (Fig. 7). With 2-3 years of growth, the plants grow large enough to be sold at significantly higher prices, providing a relatively stable cash income supply for the largely subsistence-based farmers. The same situation has been documented among the Lisu people in Nujiang, northwest Yunnan, China, who have similarly begun cultivating medicinal plants with high economic values [74].

This highlights the beginning steps of local domestication for these high-value medicinal edible plants, but this phenomenon also has implications on the biodiversity conservation as well. Essentially, there exists a significant pool of wild-collected germplasm spread out across a relatively extensive network of rural villages with specific knowledge of their provenance. In light of decimated wild populations, these household collections of wildcollected species collectively function as a germplasm bank that could potentially be tapped by conservation organizations wishing to re-establish healthy, genetically diverse, wild populations of these threatened species.

Rosa roxburghii presents another example of WEP domestication in Liangshan with conservation implications. Originally harvested from the wild as a snack, Rosa roxburghii is now widely planted in many Yi courtyards, where specimens have been selected to produce larger and more evenly maturing fruit. The market price of cultivated Rosa roxburghii fruit is now eight to ten times that of the fruit directly collected from the wild [31]. Consequently, further domestication of cultigens may also help alleviate collection pressures on some WEPs.

\section{Conclusion}

Our survey documented 105 WEPs in Liangshan Autonomous Prefecture. The traditional knowledge held about these plants is the result of the accumulated experience by the Liangshan Yi people's long-term presence living in the local environment. With the rise of functional foods and edible medicinal plants, there is a need to further analyze the nutrition, chemical composition, and bioactivity of the WEPs. For sustainable utilization, some species with high medicinal value but sharp declines in wild populations should be further studied for resource assessment, sustainable use, domestication possibilities, and genetic conservation.

\section{Acknowledgements}

We are grateful to all the field assistants (particularly Ercong Aba) and interviewees who provided information in the ethnobotany surveys in Liangshan.

\section{Authors' contributions}

All authors contributed substantively to this project, either through data collection (KA, JW), translation and analysis (BCS, JW, KA, YZ), and/or writing, revising, and editing $(B C S, J W, T T)$. The first author, JW, has been the primary researcher involved at each stage of this project, culminating in this manuscript All authors have approved this manuscript in its current and final form.

\section{Funding}

This research was funded by the Biodiversity Survey and Assessment Project of the Ministry of Ecology and Environment, China (\#2019HJ2096001006); the National Natural Science Foundation of China (\#31600253); and the China Scholarship Council (201708515018).

\section{Availability of data and materials}

The dataset supporting the conclusions of this article is included within the article (and its tables).

Ethics approval and consent to participate

The authors assert that all procedures contributing to this work comply with the applicable ethical standards of the relevant national and institutional committees on human experimentation and with the Helsinki Declaration of 1975, as revised in 2008. All interviews were conducted only after oral informed consent was obtained.

Consent for publication

Not applicable.

\section{Competing interests}

The authors declare that they have no competing interests.

\section{Author details}

'School of Architecture and Civil Engineering, Chengdu University, Chengdu 610106, China. ${ }^{2}$ Department of Environment, Sichuan University, Chengdu 610065, China. ${ }^{3}$ Department of Botany, University of Hawai'i at Mānoa, Honolulu, HI 96822, USA. ${ }^{4}$ Leibo County Youth League Committee, Jincheng Town 616550, Leibo County, China.

Received: 23 June 2019 Accepted: 5 December 2019

Published online: 26 February 2020

\section{References}

1. Hajjar R, Hodgkin T. The use of wild relatives in crop improvement: a survey of developments over the last 20 years. Euphytica. 2007;156:1-13.

2. Pandey A, Tomer AK, Bhandari DC, Pareek SK. Towards collection of wild relatives of crop plants in India. Genet Resour Crop Evol. 2008;55:187-202.

3. Maxted N, Kell S, Ford-Lloyd B, Dulloo E, Toledo A. Toward the systematic conservation of global crop wild relative diversity. Crop Sci. 2012;52:774-85.

4. Uprety Y, Poudel RC, Shrestha KK, Rajbhandary S, Tiwari NN, Shrestha UB, et al. Diversity of use and local knowledge of wild edible plant resources in Nepal. J Ethnobiol Ethnomed. 2012;8:16. 
5. Ju Y, Zhuo J, Liu B, Long C. Eating from the wild: diversity of wild edible plants used by Tibetans in Shangri-la region, Yunnan, China. J Ethnobiol Ethnomed [Internet]. 2013;9:1-22 Available from: http://www.ethnobiomed. com/content/pdf/1746-4269-9-28.pdf.

6. Shumsky S, Hickey GM, Johns T, Pelletier B, Galaty J. Institutional factors affecting wild edible plant (WEP) harvest and consumption in semi-arid Kenya. Land use policy [Internet]. Elsevier Ltd; 2013;38: 48-69. Available from: https://doi.org/10.1016/j.landusepol.2013.10.014

7. de Oliveira G, Lima-Ribeiro MS, Terribile LC, Dobrovolski R, Telles MP, de C, JAF D-F. Conservation biogeography of the Cerrado's wild edible plants under climate change: linking biotic stability with agricultural expansion. Am J Bot. 2015;102:870-7.

8. Meilleur BA, Hodgkin T. In situ conservation of crop wild relatives: status and trends. Biodivers Conserv. 2004;13:663-84

9. Etkin NL, editor. Eating on the wild side: the pharmacologic, ecologic, and social implications of using noncultigens. Tucson: University of Arizona Press; 1994.

10. Pinela J, Carvalho AM, Ferreira ICFR. Wild edible plants: nutritional and toxicological characteristics, retrieval strategies and importance for today's society. Food Chem Toxicol. Elsevier. 2017;110:165-88.

11. Bortolotto IM, de Mello Amorozo MC, Neto GG, Oldeland J, DamascenoJunior GA. Knowledge and use of wild edible plants in rural communities along Paraguay River, Pantanal, Brazil. J Ethnobiol Ethnomed [Internet]. Journal of Ethnobiology and Ethnomedicine; 2015;11. Available from: https://doi.org/10.1186/s13002-015-0026-2

12. Ford-Lloyd BV, Schmidt M, Armstrong SJ, Barazani O, Engels J, Hadas R, et al. Crop wild relatives - undervalued, underutilized and under threat? Bioscience. 2011:61:559-65.

13. Grunert KG. Food quality and safety: consumer perception and demand. Eur Rev Agric Econ. 2005;32:369-91.

14. Etkin NL. Ethnopharmocology: biobehavioral approaches in the anthropological study of indigenous medicines. Annu Rev Anthropol. 1988; 17:23-42.

15. Etkin NL. Edible medicines: an ethnopharmacology of food. Tucson: University of Arizona Press; 2006.

16. Johns T. The origins of human diet and medicine: chemical ecology. Tucson: University of Arizona Press; 1996.

17. Moerman DE. An analysis of the food plants and drug plants of native North America. J Ethnopharmacol. 1996;52:1-22.

18. Pei S, Huai H. Ethnobotany. Shanghai: Shanghai Science and Technology Press; 2007.

19. Etkin NL, Ross PJ. Food as medicine and medicine as food: an adaptive framework for the interpretation of plant utilization among the Hausa of northern Nigeria. Soc Sci Med. 1982;16:1559-73.

20. Regassa T, Kelbessa E, Asfaw Z. Ethnobotany of wild and semi-wild edible plants of Chelia District, West-Central Ethiopia. Sci Technol Arts Res J. 2014;3:122-34.

21. Ramachandran VS. Wild edible plants of the Anamalais, Coimbatore district, western Ghats. Tamil Nadu. Indian J Tradit Knowl. 2007;6:173-6.

22. Ong HG, Chung JM, Jeong HR, Kim YD, Choi $K$, Shin $\mathrm{CH}$, et al. Ethnobotany of the wild edible plants gathered in Ulleung Island, South Korea. Genet Resour Crop Evol. Springer Netherlands. 2016;63:409-27.

23. Kang Y, Łuczaj $Ł$, Kang J, Wang F, Hou J, Guo Q. Wild food plants used by the Tibetans of Gongba Valley (Zhouqu county, Gansu, China). J Ethnobiol Ethnomed. 2014;10:1-13.

24. Pieroni A, Giusti ME. Alpine ethnobotany in Italy: traditional knowledge of gastronomic and medicinal plants among the Occitans of the upper Varaita valley, Piedmont. J Ethnobiol Ethnomed. 2009;5:1-13.

25. McClatchey WC. Wild food plants of Remote Oceania. Acta Soc Bot Pol. 2012;81:371-80.

26. Gaoue OG, Coe MA, Bond M, Hart G, Seyler BC, McMillen H. Theories and major hypotheses in ethnobotany. Econ Bot. 2017;71:269-87.

27. Bradley D. Ch. 12: Language policy for the Yi. In: Harrell S, editor. Perspect Yi Southwest China. Berkeley: University of California Press; 2001. p. 195-213.

28. Wang J, Seyler BC, Ticktin T, Zeng Y, Ezhu Z. Indigenous botanical nomenclature used by the Yi people in Liangshan Prefecture, Sichuan Province, China. Econ Bot. 2019;73:325-40

29. Lawson J. A frontier made lawless: violence in Upland Southwest China, 1800-1956. Vancouver: University of British Columbia Press; 2017.

30. Qubumo B. Traditional Nuosu origin narratives: a case study of ritualized epos in Bimo incantation scriptures. Oral Tradit. 2001;2:453-79.
31. Wang J, Wang T, Qiu C, Fu L. "Liángshān zhōu yí, hàn hùn jū qū y̌̌nshí wénhuà zhōng de yěshēng zhíwù lìyòng chūtàn" 凉山州彞、汉混居区饮 食文化中的野生植物利用初探 [A study on the utilization of wild plants for food in Liangshan Yi Autonomous Prefecture]. Plant Divers Resour. 2013; 35:461-71.

32. Li L. "Yizu hei_—liangshan yizu zhiwu ran hei yanjiu" 彞族黑——凉山絭 族植物染黑研究 [Black Yi: a study of the plant-based black dyes of Liangshan's Yi People]. Beijing Institute of Fashion; 2010.

33. Wang J. "Sìchuān liángshān yízú chuántǒng mínsú zhōng de zhíwù jí qí wénhuà yìy”" 四川凉山彞族传统民俗中的植物及其文化意义 [Plants among Yi people's traditional folk customs and their cultural significance in Liangshan Yi Autonomous Prefecture Sichuan]. Plant Divers Resour. 2014;36: 537-44.

34. Phillips O, Gentry AH. The useful plants of Tambopata, Peru: II. Additional hypothesis testing in quantitative ethnobotany. Econ Bot. 1993;47:33-43.

35. Yang Y, Tian K, Hao J, Pei S, Yang Y. Biodiversity and biodiversity conservation in Yunnan, China. Biodivers Conserv [Internet]. Springer Netherlands; 2004;13:813-26. Available from: https://doi.org/10.1023/B:BIOC. $0000011728.46362 .3 \mathrm{C}$

36. Li D-Z, Pritchard HW. The science and economics of ex situ plant conservation. Trends Plant Sci [Internet]. 2009;14:614-21. Available from: http://www.sciencedirect.com/science/article/pii/S1360138509002325

37. Yin K. Liangshan Yearbook: 2016 Edition. Historical Records Office Liangshan Yi Autonomous Prefecture, editor. Chinese Literature and History Press; 2017.

38. Bender M. "Tribes of snow": animals and plants in the Nuosu "Book of Origins." Asian Ethnol. 2008;67:5-42.

39. Liangshan Statistics Bureau. Statistical Yearbook of Liangshan: 2016 Edition [Internet]. 2016 [cited 2019 Jan 15]. Available from: http://tjj.Isz.gov.cn/ztjj/ tinj(2016)97/5257581/index.shtml

40. Sichuan Investment Promotion Bureau. Liangshan Prefecture [Internet]. People's Gov. Sichuan Prov. 2014 [cited 2019 Jan 15]. Available from: http:// www.sc.gov.cn/10462/10580/10587/2014/9/22/10313925.shtml

41. Lama ZQ-F. Subgrouping of Nisoic (Yi) languages: a study from the perspectives of shared innovation and phylogenetic estimation. University of Texas at Arlington; 2012.

42. Ma H. "Yiyu cifa yanjiu" 璘语词法研究 [Yi Language lexical research]. Shanghai Normal University; 2012.

43. Bradley D. Tibeto-Burman languages and classification. In: Bradley D, editor. Pap Southeast Asian Linguist 14 Tibet Lang Himalayas. Canberra: Australian Natonal University; 1997. p. 1-72.

44. He Y. "Yi Han da cidian" 彞汉大词典. [Yi-Han Chinese dictionary]. Chengdu: Sichuan Publishing Group, Sichuan Nationalities Publishing House; 2008.

45. Jiari M. "Wăn qīng yúnnán xiǎo liángshān yízú qiānxǐ chuánshuō zhōng de zú jì guānxì yǔ qūyù zhèngzhì" 晚清云南小凉山彞族迁徙传说中的族际关 系与区域政治 [Inter-ethnic relations and regional politics in the legend of the migration of the Xiaoliangshan Dais in Yunnan in the Late Qing Dynast. J Southwest Minzu Univ (Humanities Soc Sci. 2018;2:62-72.

46. Harrell S. Ethnicity, local interests, and the state: Yi communities in Southwest China. Comp Stud Soc Hist. 1990;32:515-48.

47. Harrell S. Introduction. In: Harrell S, editor. Perspect Yi Southwest China. Berkeley: University of California Press; 2001. p. 1-17.

48. Mao G. "Găigé kāifàng y̌llái yízú zōngjiào yánjiū zòngshù" 改革开放以来彞 族宗教研究综述 [A review of Yi religion scholarship since the reform and opening up]. Econ Res Guid. 2014;222:7-9.

49. Bernard HR. Research methods in anthropology: qualitative and quantitative approaches. 5th ed. Lanham, MD: AltaMira Press; 2011.

50. Alexiades MN. Collecting ethnobotanical data: an introduction to basic concepts and techniques. In: Alexiades MN, Sheldon JW, editors. Sel Guidel Ethnobot Res A F Man. Bronx, New York: The New York Botanical Garden; 1996. p. 58-94.

51. Balick MJ, Cox P. Plants, people, and culture: the science of ethnobotany. New York: Scientific American Library; 1996.

52. Phillips O, Gentry AH. The useful plants of Tambopata, Peru: I. Statistical hypotheses tests with a new quantitative technique. Econ Bot. 1993;47: 15-32.

53. Zeng $W$, Zhao T. Preliminary study on the optimal pollination period of Paris polyphylla var. yunnanensis. Med Plant. 2012;3:8-10.

54. Zhang S, Yang Z, Huang Z, Yu M, Liu Y, Zhang H. Quality evaluation of polygerm Paris polyphylla var. yunnanensis (Polygerm Varieties) in Yunnan Province Based on Moisture, Ashes and Extracts. Med Plant. 2016;7:24-8. 
55. Liu T, Greenslade A, Yang S. Levels of rhizome endophytic fungi fluctuate in Paris polyphylla var. yunnanensis as plants age. Plant Divers. 2017;39:60-4.

56. Ding L, Zhao M, Li Y-M, Chen L, Wang Z, Wang Z, et al. Study on the antinociceptive and anti-inflammatory effects of the extract of aerial part and rhizome of Paris polyphylla var. chinensis. Nat Prod Res Dev. 2018;30:832-9.

57. Wang M. "Liangshan Yizu kouchuan wenhua baohu fazhan kunjing yu duice yanjiu" 凉山彞族口传文化保护发展困境与对策研究 [Study on the dilemma and countermeasures of Liangshan Yi oral culture protection and development]. J Southwest Minzu Univ (Humanities Soc Sci. 2015;36:51-7.

58. Sõukand R, Pieroni A, Biró $M$, Dénes $A$, Dogan $Y$, Hajdari A, et al. An ethnobotanical perspective on traditional fermented plant foods and beverages in Eastern Europe. J Ethnopharmacol. 2015;170:284-96.

59. Katz SE. The art of fermentation: an in-depth exploration of essentia concepts and processes from around the world. White River Junction, VT: Chelsea Green Publishing; 2012.

60. Bibalani GH, Mosazadeh-Sayadmahaleh F. Recognition and consumption uses and medicinal properties of sour orange (Citrus aurantium) by rural people in east part of Gilan Province (North Iran). J Med Plants Res. 2011;5: 1238-43.

61. Shuo G. "J̄ yǔ yízú chuántǒng wénhuà" 鸡与彞族传统文化 [Chicken and Yi traditional culture]. Guizhou Ethn Stud. 1994;57:65-70.

62. He J, Zhang R, Lei Q, Chen G, Li K, Ahmed S, et al. Diversity, knowledge, and valuation of plants used as fermentation starters for traditional glutinous rice wine by Dong communities in Southeast Guizhou, China. J Ethnobiol Ethnomed [Internet]; 2019;15:10. Available from: https:/ethnobiomed. biomedcentral.com/articles/10.1186/s13002-019-0299-y

63. Alencar NL, de Sousa Araújo TA, de Amorim ELC, de Albuquerque UP. The inclusion and selection of medicinal plants in traditional pharmacopoeiasEvidence in support of the diversification hypothesis. Econ Bot. 2010;64:68-79.

64. Cristancho S, Vining J. Culturally defined keystone species. Hum Ecol Rev. 2004;11:153-64.

65. Garibaldi A, Turner N. Cultural keystone species: implications for ecological conservation and restoration. Ecol Soc. 2004;9:1.

66. Hoffman B, Gallaher T. Importance indices in quantitative ethnobotany. Ethnobot Res Appl. 2007;5:201-18.

67. Lv Z, Lan Y. Cultural implication of Liangshan Yi in clothing design. Guizhou Ethn Stud. 2014:35:150-3.

68. Zhu G, Xu J. "Cóng guizhōu yízú yīxué wénxiàn 'qĩ gǔ shǔ' kàn yízú 'yīyào' wénhuà tèsè" 从贵州彝族医学文献《启谷署》看率族 “医药” 文化特色

The cultural characteristics of Yi medicine from the medical literature: Qi Gushu]. J Sichuan Minzu Coll. 2015;24:30-4.

69. Li L, Li L, Li D. "Láncāngjiāng liúyù yízú yĩyào wénhuà yánjiü -xīshuāngbănnà měnglà xiàn xiàng míng xiāng yízú yīyào diàochá" 澜沧江流域彝族医药文化研究———西双版纳预腊县象明乡彞族医药 调查 [Studies on medicine of Yi people in Lancang River Region]. J Baoshan Univ. 2011:6:64-8.

70. Liu Y, Long C, Dao Z. "Yúnnán sīmáo duānwǔ jiéshí yòngyào gēn de mínzú zhíwù xué diàochá" 云南思茅端午节食用药根的民族植物学调查 [Ethnobotanical survey on medicinal roots eaten by the local people in Simao, Yunnan Province, during the Dragon-Boat Festivall]. J Plant Resour Environ. 2003;12:33-8.

71. McCune LM, Johns T. Antioxidant activity relates to plant part, life form and growing condition in some diabetes remedies. J Ethnopharmacol. 2007;112:461-9.

72. Long C, Li S, Long B, Shi Y, Liu B. Medicinal plants used by the Yi ethnic group: a case study in central Yunnan. J Ethnobiol Ethnomed. 2009:5:1-5.

73. Wu Y. "Mínzú yīyào wénhuà chănyè shēngcún huánjìng yŭ fãzhăn lùjìng tànsuǒ —y̌̃ Sichuān mínzú diqū wéi lì" 民族医药文化产业 生存环境与发 展路径探索 ——— - 以四川民族地区为例 [Exploration on survival environment and development path of ethnic medicinal culture industry -case study on the Sichu. J Southwest Minzu Univ (Humanities Soc Sci. 2015; 8:141-6.

74. Huang J, Pei S, Long C. An ethnobotanical study of medicinal plants used by the Lisu people in Nujiang, Northwest Yunnan, China. Econ Bot [Internet]. Springer on behalf of New York Botanical Garden Press; 2004;58: S253-64. Available from: http://www.jstor.org/stable/4256922

\section{Publisher's Note}

Springer Nature remains neutral with regard to jurisdictional claims in published maps and institutional affiliations.

Ready to submit your research? Choose BMC and benefit from:

- fast, convenient online submission

- thorough peer review by experienced researchers in your field

- rapid publication on acceptance

- support for research data, including large and complex data types

- gold Open Access which fosters wider collaboration and increased citations

- maximum visibility for your research: over $100 \mathrm{M}$ website views per year

At BMC, research is always in progress.

Learn more biomedcentral.com/submissions 